\title{
A COMBINATORIAL APPROACH TO NONINVOLUTIVE SET-THEORETIC SOLUTIONS OF THE YANG-BAXTER EQUATION
}

\author{
TATIANA GATEVA-IVANOVA
}

\begin{abstract}
We study noninvolutive set-theoretic solutions $(X, r)$ of the YangBaxter equations in terms of the properties of the canonically associated algebraic objects-the braided monoid $S(X, r)$, the quadratic Yang-Baxter algebra $A=A(\mathbf{k}, X, r)$ over a field $\mathbf{k}$ and its Koszul dual, $A$ !. More generally, we continue our systematic study of nondegenerate quadratic sets $(X, r)$ and the associated quadratic algebras $A=A(\mathbf{k}, X, r)$ and the Koszul dual $A^{!}$. Next we investigate the class of (noninvolutive) square-free solutions $(X, r)$. It contains the special class of self distributive solutions (quandles). We make a detailed characterization in terms of various algebraic and combinatorial properties each of which shows the contrast between involutive and noninvolutive square-free solutions. We introduce and study a class of finite square-free braided sets $(X, r)$ of order $n \geq 3$ which satisfy "the minimality condition M", that is $\operatorname{dim}_{\mathbf{k}} A_{2}=2 n-1$. Examples are some simple racks of prime order $p$. Finally, we discuss general extensions of solutions and introduce the notion of a generalized strong twisted union of braided sets. We prove that if $(Z, r)$ is a non-degenerate 2-cancellative braided set splitting as $Z=X \natural^{*} Y$, where $X$ and $Y$ are $r$-invariant subsets then its braided monoid $S_{Z}$ is a generalized strong twisted union $S_{Z}=S_{X}$ 七* $^{*} S_{Y}$ of the braided monoids $S_{X}$ and $S_{Y}$. Moreover, if $(Z, r)$ is injective then its braided group $G_{Z}=G(Z, r)$ also splits as $G_{Z}=G_{X} \natural^{*} G_{Y}$ of the associated braided groups of $X$ and $Y$. We propose a construction of a generalized strong twisted union $Z=X \natural^{*} Y$ of braided sets $\left(X, r_{X}\right)$, and $\left(Y, r_{Y}\right)$, where the map $r$ has high, explicitly prescribed order.
\end{abstract}

\section{Contents}

1. Introduction

2. Preliminaries

3. Nondegenerate quadratic sets with 2-cancellation and their quadratic algebras

4. Square-free quadratic sets with cyclic conditions

5. Square-free braided sets and the contrast between the involutive and noninvolutive cases

6. Square-free braided sets $(X, r)$ satisfying the minimality condition 24

7. A class of special extensions 32

8. The braided monoid $S(X, r)$ and extensions of solutions 35

Date: October 7, 2019.

1991 Mathematics Subject Classification. Primary 81R50, 16W50, 16S36, 16 S37.

Key words and phrases. Yang-Baxter, Braided sets, Quadratic sets, Quadratic algebras, Koszul algebras.

The author was partially supported by The Abdus Salam International Centre for Theoretical Physics (ICTP), Trieste, and by The Max Planck Institute for Mathematics (MPIM), Bonn. 


\section{INTRODUCTION}

It was established in the last three decades that solutions of the linear braid or Yang-Baxter equations (YBE) on a vector space of the form $V^{\otimes 3}$ lead to remarkable algebraic structures. Here $r: V \otimes V \longrightarrow V \otimes V, r^{12}=r \otimes \mathrm{id}, r^{23}=i d \otimes r$ is a notation and structures include coquasitriangular bialgebras $A(r)$, their quantum group (Hopf algebra) quotients, quantum planes and associated objects, at least in the case of specific standard solutions, see [26, 27]. On the other hand, the variety of all solutions on vector spaces of a given dimension has remained rather elusive in any degree of generality. It was proposed by V.G. Drinfeld [9], to consider the same equations in the category of sets, and in this setting numerous results were found. It is clear that a set-theoretic solution extends to a linear one, but more important than this is that set-theoretic solutions lead to their own remarkable algebraic and combinatoric structures, only somewhat analogous to quantum group constructions. In the present paper we continue our systematic study of set-theoretic solutions based on the associated quadratic algebras and monoids that they generate.

More generally, we study quadratic sets and their algebraic objects. The notions of a quadratic set $(X, r)$ and its related algebraic objects were introduced by the author and studied first in [13, see also [19, 15] for more results on quadratic sets $(X, r)$. We shall use the terminology, notation and some results from [13, 15, 18, 17. 19.

Definition 1.1. [13] Let $X$ be a nonempty set (possibly infinite) and let $r: X \times$ $X \longrightarrow X \times X$ be a bijective map. In this case we use notation $(X, r)$ and refer to it as a quadratic set. The image of $(x, y)$ under $r$ is presented as

$$
r(x, y)=\left({ }^{x} y, x^{y}\right) .
$$

This formula defines a "left action" $\mathcal{L}: X \times X \longrightarrow X$, and a "right action" $\mathcal{R}$ : $X \times X \longrightarrow X$, on $X$ as: $\mathcal{L}_{x}(y)={ }^{x} y, \mathcal{R}_{y}(x)=x^{y}$, for all $x, y \in X$. (i) $r$ is nondegenerate, if the maps $\mathcal{L}_{x}$ and $\mathcal{R}_{x}$ are bijective for each $x \in X$. (ii) $r$ is involutive if $r^{2}=i d_{X \times X}$. (iii) $(X, r)$ is square-free if $r(x, x)=(x, x)$ for all $x \in X$. (iv) $(X, r)$ is quantum binomial if it is nondegenerate, square-free, and involutive. (v) $r$ is $a$ set-theoretic solution of the Yang-Baxter equation (YBE) if the braid relation

$$
r^{12} r^{23} r^{12}=r^{23} r^{12} r^{23}
$$

holds in $X \times X \times X$, where $r^{12}=r \times \mathrm{id}_{X}$, and $r^{23}=\mathrm{id}_{X} \times r$. In this case we refer to $(X, r)$ also as a braided set. A braided set $(X, r)$ with $r$ involutive is called $a$ symmetric set.

In this paper we always assume that $r$ is nondegenerate. Also, as a notational tool, we shall often identify the sets $X^{\times m}$ and $X^{m}$, the set of all monomials of length $m, m \geq 2$, in the free monoid $\langle X\rangle$.

As in our previous works $([13,15,[18,[17,19])$ to each quadratic set $(X, r)$ we associate canonically several algebraic objects (see Definition 2.4) generated by $X$ and with quadratic defining relations naturally determined as

$$
x y=y^{\prime} x^{\prime} \in \Re(r) \text { iff } r(x, y)=\left(y^{\prime}, x^{\prime}\right) \text { and }(x, y) \neq\left(y^{\prime}, x^{\prime}\right) \text { hold in } X \times X .
$$


Note that in the case when $\mathrm{X}$ is finite, the set $\Re(r)$ of defining relations is also finite, therefore the associated algebraic objects are finitely presented.

In this paper we continue our systematic study of the close relations between the combinatorial properties of the defining relations, i.e. of the map $r$, and the structural properties of the associated algebraic objects.

In the first half of the paper we investigate non-degenerate quadratic sets $(X, r)$ of finite order, their quadratic graded algebras $A$, and the monoid $S(X, r)$. Section 2 contains preliminary material on quadratic sets, their quadratic algebras and monoids. In Section 3 we study non-degenerate quadratic sets $(X, r)$, with 2cancellation. Proposition 3.9 provides upper and lower bounds for the dimension $\operatorname{dim} A_{2}$, and shows that the upper bound is attained, whenever $r$ is involutive. The main result of the section is Theorem 3.16. It implies, in particular, that a squarefree non-degenerate quadratic set $(X, r)$, with $|X|=n$ is a symmetric set if and only if its quadratic algebra $A$ has Hilbert series $H_{A}(z)=\frac{1}{(1-z)^{n}}$. The theorem improves an old result of the author, see [16], Theorem 2. In Section 4 we pay special attention to square-free quadratic sets with cyclic conditions. We find some new combinatorial results, see Theorem 4.7 and use them to show that, surprisingly, a square-free quadratic set $(X, r)$ of finite order $|X|=n$ which satisfies the cyclic conditions is a symmetric set if and only if $\operatorname{dim}_{\mathbf{k}} A_{3}^{!}=\left(\begin{array}{l}n \\ 3\end{array}\right)$, see Proposition 4.8 In Section 5 we study square-free braided sets and the contrast between the involutive and noninvolutive cases. We show that every square-free braided set (of arbitrary cardinality) satisfies the cyclic conditions. Theorem [5.5 characterizes the involutive braided sets $(X, r)$ in terms of various equivalent properties of the algebra $A$, its Koszul dual $A^{!}$and the monoid $S(X, r)$. Corollary 5.6 provides a characterization of noninvolutive square-free braided sets. In Section $[$ we introduce quadratic sets $(X, r)$ which satisfy "the minimality condition", $\mathbf{M}$, that is $\operatorname{dim}_{\mathbf{k}} A_{2}=2 n-1$, see Definition 6.1] We first investigate (general) square-free 2-cancellative quadratic sets $(X, r)$ with minimality condition and prove Proposition 6.5. We make some initial steps in the study of braided sets, and in particular, quandles with minimality condition, M. Corollary 6.18 implies that every square-free self distributive solution, $(X, r)$ (see Definition [6.8), corresponding to a dihedral quandle of prime order $|X|=p>2$ satisfies the minimality condition $\mathbf{M}$. We end up the section with a list of questions which have stimulated recent results of other authors. In Sec. 7 we propose a construction which generates noninvolutive extensions $(Z, r)$ of braided (or symmetric) sets, where the map $r$ has high, explicitly prescribed order, see Theorem 7.2. Finally, in Section 8 are studied braided monoids $S(X, r)$ and extensions of solutions. We consider "general" extensions of braided sets. In Subsec. 8.4 we introduce "generalized strong twisted unions $Z=X \natural^{*} Y$ of non-degenerate braided sets", see Definition 8.7 The main result of the section is Theorem 8.12 .

\section{Preliminaries}

During the last two decades the study of set-theoretic solutions of the Yang-

Baxter equation and related structures notably intensified, a relevant selection of works for the interested reader is $99,21,10,25,13,4,33,36,19,15,16,18,17,4$, 37, 23, 24, 34, 35, et all. In this section we recall basic notions and results which will be used in the paper. We shall use the terminology, notation and some results from [13, 15, 18, 17, 19. 
Remark 2.1. Let $(X, r)$ be a quadratic set, and let ${ }^{x} \bullet$, and $\bullet^{x}$ be the associated left and right actions. Then

(1) The map $r$ is involutive iff the actions satisfy:

$$
{ }^{u} v\left(u^{v}\right)=u \text {, and }\left({ }^{u} v\right)^{u^{v}}=v, \forall u, v \in X .
$$

(2) $r$ is square-free if and only if ${ }^{x} x=x$, and $x^{x}=x, \forall x \in X$.

(3) If $r$ is nondegenerate and square-free, then

$$
\begin{aligned}
& { }^{z} t={ }^{z} u \quad \Longleftrightarrow t=u \Longleftrightarrow t^{z}=u^{z} \\
& { }^{z} t=z \Longleftrightarrow t=z \Longleftrightarrow t^{z}=z .
\end{aligned}
$$

Remark 2.2. [10] Let $(X, r)$ be quadratic set. Then $r$ obeys the YBE, that is $(X, r)$ is a braided set iff the following conditions hold for all $x, y, z \in X$ :

$$
\begin{gathered}
\text { 11: } \left.\quad{ }^{x}\left({ }^{y} z\right)={ }^{x} y\left(x^{y} z\right), \quad \mathbf{r} 1: \quad\left(x^{y}\right)^{z}=\left(x^{y} z\right)\right)^{y^{z}}, \\
\left.\operatorname{lr3}: \quad\left({ }^{x} y\right)^{\left(x^{y} z\right)}={ }^{\left(x^{y} z\right.}\right)\left(y^{z}\right) .
\end{gathered}
$$

Convention 2.3. In this paper by "a solution" we mean "a non-degenerate braided set" $(X, r)$, where $X$ is a set of arbitrary cardinality. We shall also refer to it as "a braided set", keeping the convention that we consider only non-degenerate braided sets. "An involutive solution" means "a non-degenerate symmetric set". In most cases we shall also assume that $r$ is 2-cancellative but this will be indicated explicitly.

2.1. Quadratic sets and their algebraic objects. Let $X$ be a non-empty set, and let $\mathbf{k}$ be a field. We denote by $\langle X\rangle$, and ${ }_{g r}\langle X\rangle$, respectively the free monoid, and the free group generated by $X$, and by $\mathbf{k}\langle X\rangle$ - the free associative $\mathbf{k}$-algebra generated by $X$. For a set $F \subseteq \mathbf{k}\langle X\rangle,(F)$ denotes the two sided ideal of $\mathbf{k}\langle X\rangle$, generated by $F$. As a notational tool, we shall often identify the sets $X^{\times m}$ of ordered $m$-tuples, $m \geq 2$, and $X^{m}$, the set of all monomials of length $m$ in the free monoid $\langle X\rangle$. For $m \geq 1$, the length of a monomial $u=x_{1} \cdots x_{m} \in X^{m}$ will be denoted by $|u|=m$.

As in our works [13, 14, 15, 19, 20, 17, we use the following.

Definition 2.4. To each quadratic set $(X, r)$ we associate canonically algebraic objects generated by $X$ and with quadratic relations $\Re=\Re(r)$ naturally determined as

$$
x y=y^{\prime} x^{\prime} \in \Re(r) \text { iff } r(x, y)=\left(y^{\prime}, x^{\prime}\right) \text { and }(x, y) \neq\left(y^{\prime}, x^{\prime}\right) \text { hold in } X \times X .
$$

The monoid $S=S(X, r)=\langle X ; \Re(r)\rangle$ with a set of generators $X$ and a set of defining relations $\Re(r)$ is called the monoid associated with $(X, r)$. The group $G=G(X, r)=G_{X}$ associated with $(X, r)$ is defined analogously.

For an arbitrary fixed field $\mathbf{k}$, the $\mathbf{k}$-algebra associated with $(X, r)$ is defined as

$$
\begin{gathered}
A=A(\mathbf{k}, X, r)=\mathbf{k}\langle X\rangle /\left(\Re_{0}\right) \simeq \mathbf{k}\langle X ; \Re(r)\rangle, \text { where } \\
\Re_{0}=\Re_{0}(r)=\left\{x y-y^{\prime} x^{\prime} \mid x y=y^{\prime} x^{\prime} \in \Re(r)\right\} .
\end{gathered}
$$

Clearly, the quadratic algebra $A$ generated by $X$ and with defining relations $\Re_{0}(r)$ is isomorphic to the monoid algebra $\mathbf{k} S(X, r)$.

Definition 2.5. We shall call a quadratic set $(X, r)$ injective if the set $X$ is embedded in $G(X, r)$. 
Recall that when $(X, r)$ is a braided set its monoid $S=S(X, r)$ is a graded braided monoid, 19], and the group $G(X, r)$ is a braided group, 25], see details in section 8. Moreover, the associated quadratic algebra $\mathrm{A}=\mathrm{A}(\mathbf{k}, \mathrm{X}, \mathrm{r})$ is also called an Yang-Baxter algebra, see [29].

Remark 2.6. [15], Proposition 2.3., If $(X, r)$ is a nondegenerate and involutive quadratic set of finite order $|X|=n$ then the set $\Re(r)$ consists of precisely $\left(\begin{array}{l}n \\ 2\end{array}\right)$ quadratic relations. Clearly, in in this case the associated algebra $A=A(\mathbf{k}, X, r)$ satisfies

$$
\operatorname{dim} A_{2}=\left(\begin{array}{c}
n+1 \\
2
\end{array}\right)
$$

Various equivalent conditions are given in Proposition 3.9.

Remark 2.7. Suppose $(X, r)$ is a finite quadratic set. Then $A$ is a quadratic algebra, generated by $X$ and with quadratic defining relations $\Re(r)$. Clearly, $A$ is a connected graded $\mathbf{k}$-algebra (naturally graded by length), $A=\bigoplus_{i \geq 0} A_{i}$, where $A_{0}=\mathbf{k}, A$ is generated by $A_{1}=\operatorname{Span}_{\mathbf{k}} X$, so each graded component $A_{i}$ is finite dimensional. Moreover, the associated monoid $S=S(X, r)$ is naturally graded by length:

$$
S=\bigsqcup_{m \geq 0} S_{m} ; \text { where } S_{0}=1, S_{1}=X, S_{m}=\{u \in S|| u \mid=m\}, S_{m} . S_{t} \subseteq S_{m+t} .
$$

In the sequel, by "a graded monoid, $S$ ", we shall mean that $S$ is generated by $S^{1}=X$ and graded by length. The grading of $S$ induces a canonical grading of its monoidal algebra $\mathbf{k} S(X, r)$. The isomorphism $A \cong \mathbf{k} S(X, r)$ agrees with the canonical gradings, so there is an isomorphism of vector spaces $A_{m} \cong \operatorname{Span}_{\mathbf{k}} S_{m}$.

Remark 2.8. [14] Let $(X, r)$ be a quadratic set, let $S=S(X, r)$ be the associated monoid.

(i) By definition, two monomials $w, w^{\prime} \in\langle X\rangle$ are equal in $S$ iff $w$ can be transformed to $w^{\prime}$ by a finite sequence of replacements each of the form

$$
a x y b \longrightarrow a r(x y) b, \text { or } a x y b \longrightarrow a r^{-1}(x y) b, \quad \text { where } x, y \in X, a, b \in\langle X\rangle \text {. }
$$

Clearly, every such replacement preserves monomial length, which therefore descends to $S(X, r)$. Furthermore, replacements coming from the defining relations are possible only on monomials of length $\geq 2$, hence $X \subset S(X, r)$ is an inclusion. For monomials of length $2, x y=z t$ holds in $S(X, r)$ iff $z t=r^{k}(x y)$ is an equality of words in $X^{2}$ for some $k \in \mathbb{Z}$.

(ii) It is convenient for each $m \geq 2$ to refer to the subgroup $D_{m}$ of the symmetric group $\operatorname{Sym}\left(X^{m}\right)$ generated concretely by the maps

$$
r^{i i+1}: X^{m} \longrightarrow X^{m}, r^{i i+1}=\mathrm{id}_{X^{i-1}} \times r \times \mathrm{id}_{X^{m-i-1}}, i=1, \cdots, m-1 .
$$

One can also consider the free groups

$$
\mathcal{D}_{m}(r)={ }_{\text {gr }}\left\langle r^{i i+1} \mid i=1, \cdots, m-1\right\rangle,
$$

where the $r^{i i+1}$ are treated as abstract symbols, as well as various quotients depending on the further type of $r$ of interest. These free groups and their quotients act on $X^{m}$ via the actual maps $r^{i i+1}$ so that the image of $\mathcal{D}_{m}(r)$ in $\operatorname{Sym}\left(X^{m}\right)$ is $D_{m}(r)$. In particular, $D_{2}(r)=\langle r\rangle \subset \operatorname{Sym}\left(X^{2}\right)$ is the cyclic group generated by $r$. It follows straightforwardly from part (i) that $w, w^{\prime} \in\langle X\rangle$ are equal as words in $S(X, r)$ iff they have the same length, say $m$, and belong to the same orbit of $\mathcal{D}_{m}(r)$ in $X^{m}$. Clearly, in this case the equality $w=w^{\prime}$ holds in the group $G(X, r)$ and in the algebra $A(\mathbf{k}, X, r)$. 
An effective part of our combinatorial approach is the exploration of the actions of the groups $\mathcal{D}_{2}(r)=\langle r\rangle$ on $X^{2}$, the group $\mathcal{D}_{3}(r)={ }_{\operatorname{gr}}\left\langle r^{12}, r^{23}\right\rangle$ on $X^{3}$, and in particular, the properties of the corresponding orbits. In the literature a $\mathcal{D}_{2}(r)$-orbit $\mathcal{O}$ in $X^{2}$ is often called "an $r$-orbit" and we shall use this terminology.

If $r$ is involutive, the bijective maps $r^{12}$ and $r^{23}$ are involutive as well, so in this case $\mathcal{D}_{3}(r)$ is the infinite dihedral group,

$$
\mathcal{D}_{3}(r)=\mathcal{D}(r)={ }_{\text {gr }}\left\langle r^{12}, r^{23} \mid\left(r^{12}\right)^{2}=e, \quad\left(r^{23}\right)^{2}=e\right\rangle .
$$

Remark 2.9. In notation and assumption as above, let $(X, r)$ be a finite quadratic set, $S=S(X, r)$ graded by length. Then the order of $S_{2}$ equals the number of $\mathcal{D}_{2}(r)$-orbits in $X^{2}$.

For positive integers $i<n$, the maps $r^{i i+1}: X^{n} \longrightarrow X^{n}$ are defined by (2.3). Recall that the braid group $B_{n}$ is generated by elements $b_{i}, 1=i=n-1$, with defining relations

$$
b_{i} b_{j}=b_{j} b_{i},|i-j|>1, \quad b_{i} b_{i+1} b_{i}=b_{i+1} b_{i} b_{i+1},
$$

and the symmetric group $S_{n}$ is the quotient of $B_{n}$ by the relations $b_{i}^{2}=1$. It is well known (and straightforward) that for every $n \geq 3$ the following hold. (i) The assignment $b_{i} \mapsto r^{i i+1}$ extends to a (left) action of $B_{n}$ on $X^{n}$ if and only if $(X, r)$ is a braided set. (ii) The assignment $b_{i} \mapsto r^{i i+1}$ extends to an action of $S_{n}$ on $X^{n}$ if and only if $(X, r)$ is a symmetric set.

\section{Nondegenerate Quadratic Sets With 2-CANCELlation And Their QUADRATIC ALGEBRAS}

3.1. Basics on quadratic algebras. Our main reference for this subsection is 31 .

A quadratic algebra is an associative graded algebra $A=\bigoplus_{i \geq 0} A_{i}$ over a ground field $\mathbf{k}$ determined by a vector space of generators $V=A_{1}$ and a subspace of homogeneous quadratic relations $R=R(A) \subset V \otimes V$. We assume that $A$ is finitely generated, so $\operatorname{dim} A_{1}<\infty$. Thus $A=T(V) /(R)$ inherits its grading from the tensor algebra $T(V)$. The Koszul dual algebra of $A$, denoted by $A^{!}$is the quadratic algebra $T\left(V^{*}\right) /\left(R^{\perp}\right)$, see [28, 29]. The algebra $A^{!}$is also referred to as the quadratic dual algebra to a quadratic algebra $A$, see [31, p.6.

Following the classical tradition (and a recent trend), we take a combinatorial approach to study $A$. The properties of $A$ will be read off a presentation $A=$ $\mathbf{k}\langle X\rangle /(\Re)$, where by convention $X$ is a fixed finite set of generators of degree 1 , $|X|=n, \mathbf{k}\langle X\rangle$ is the unital free associative algebra generated by $X$, and $(\Re)$ is the two-sided ideal of relations, generated by a finite set $\Re$ of homogeneous polynomials of degree two. Clearly $A$ is a connected graded k-algebra (naturally graded by length) $A=\bigoplus_{i \geq 0} A_{i}$, where $A_{0}=\mathbf{k}, A$ is generated by $A_{1}=\operatorname{Span}_{\mathbf{k}} X$, and each $A_{i}$ is finite dimensional.

A quadratic algebra $A$ is a $P B W$ algebra if there exists an enumeration of $X$, $X=\left\{x_{1}, \cdots x_{n}\right\}$ such that the quadratic relations $\Re$ form a (noncommutative) Gröbner basis with respect to the degree-lexicographic ordering $<$ on $\langle X\rangle$ extending $x_{1}<x_{2}<\cdots<x_{n}$. In this case the set of normal monomials $(\bmod \Re)$ forms a $\mathbf{k}$ basis of $A$ called a $P B W$ basis and $x_{1}, \cdots, x_{n}$ (taken exactly with this enumeration) are called $P B W$-generators of $A$. The notion of a $P B W$ algebra was introduced by 
Priddy, 32. His $P B W$ basis is a generalization of the classical Poincaré-BirkhoffWitt basis for the universal enveloping of a finite dimensional Lie algebra. PBW algebras form an important class of Koszul algebras. The interested reader can find information on quadratic algebras and, in particular, on Koszul algebras and PBW algebras in 31.

There are various equivalent definitions of a Koszul algebra, see for example [31, p. 19, we recall one of them. A graded $\mathbf{k}$-algebra $A$ is Koszul if $A$ is quadratic and $\operatorname{Ext}_{A}^{*}(\mathbf{k}, \mathbf{k}) \simeq A^{!}$. It is known that if $(X, r)$ is a finite square-free involutive solution then its quadratic algebra $A(\mathbf{k}, X, r)$ is Koszul, see [21. We shall prove that, conversely, if $(X, r)$ is a (general) square-free nondegenerate braided set and its algebra $A(\mathbf{k}, X, r)$ is Koszul, then $r$ is involutive. This follows from our more general result, Proposition 3.12 .

The following results can be used to test whether a quadratic algebra is Koszul.

Fact 3.1. (1) (Theorem of S. Priddy, 32.) Every quadratic PBW algebra is Koszul.

(2) (31], Corollary 2.22.) If $A$ is a quadratic Koszul algebra, with Koszul dual $A^{!}$, then their Hilbert series satisfy

$$
H_{A}(z) \cdot H_{A^{!}}(-z)=1 \text {. }
$$

Note that (3.1) is a necessary but not a sufficient condition for Koszulity of A, 31.

3.2. Quadratic set with 2-cancellation and their quadratic algebras. To proceed further, we require some cancellation conditions.

Definition 3.2. 19], Def.2.10. A quadratic set $(X, r)$ is 2-cancellative if for every positive integer $k$, less than the order of $r$, the following two conditions hold:

$$
r^{k}(x, y)=(x, z) \Longrightarrow z=y, \quad r^{k}(x, y)=(t, y) \Longrightarrow x=t .
$$

It is obvious that the monoid $S=S(X, r)$ has cancelation on monomials of length 2 if and only if $r$ is 2-cancellative. Moreover, every injective quadratic set $(X, r)$ (see Definition 2.5) is 2-cancellative, . Note that if $x, y, z \in X, y \neq z$ each of the equalities $r^{k}(x, y)=(x, z)$, or $r^{k}(y, x)=(z, x)$ implies $y=z$ in $G(X, r)$.

Remark 3.3. (1) Every nondegenerate involutive quadratic set $(X, r)$ is 2-cancellative, see [19], Corollary 2.13. Recall that when $X$ is a finite (nondegenerate) symmetric set the monoid $S=S(X, r)$ is embedded in the group $G(X, r)$ and therefore $S$ is a monoid with cancellation. (2) A non-degenerate braided set $(X, r)$ may fail to be 2-cancellative, see Example 3.2.1 (3) There exist various (noninvolutive) non-degenerate braided sets $(X, r)$, where $r$ is 2-cancellative but the corresponding monoid $S(X, r)$ fails to be 3 -cancellative.

We shall prove that if $(X, r)$ is a finite square-free braided set, then the monoid $S(X, r)$ is cancellative iff $r$ is involutive, see Proposition 5.4 .

Notation 3.4. Denote by $\Delta_{m}$ the diagonal of $X^{\times m}, m \geq 2$ :

$$
\Delta_{m}:=\operatorname{diag}\left(X^{m}\right)=\left\{x^{m} \mid x \in X\right\} .
$$

One has $\Delta_{3}=\left(\Delta_{2} \times X\right) \bigcap\left(X \times \Delta_{2}\right)$.

Notation 3.5. Suppose $(X, r)$ is a quadratic set. The element $(x, y) \in X^{2}$ is $r$-fixed if $r(x, y)=(x, y)$. The set of $r$-fixed points in $X^{2}$ will be denoted by $\mathcal{F}(X, r)$,

$$
\mathcal{F}(X, r)=\left\{x y \in X^{2} \mid r(x, y)=(x, y)\right\}
$$


Examples 3.2.1. $\quad$ (a) Let $X=\{x, y, z\}$, and let $\rho=(x y z)$, be a cycle of length three in $\operatorname{Sym}(X)$. Define $r(a, b):=(\rho(b), a)$. Then $r: X \times X \longrightarrow X \times X$ is a non-degenerate bijection of order 6 .

$$
\begin{aligned}
& (x, x) \longrightarrow^{r}(y, x) \longrightarrow^{r}(y, y) \longrightarrow^{r}(z, y) \longrightarrow^{r}(z, z) \longrightarrow^{r}(x, z) \longrightarrow^{r}(x, x), \\
& (x, y) \longrightarrow^{r}(z, x) \longrightarrow^{r}(y, z) \longrightarrow^{r}(x, y) .
\end{aligned}
$$

It is easy to check that $(X, r)$ is a non-degenerate braided set (this is a permutation solution). The actions satisfy: $\mathcal{L}_{x}=\mathcal{L}_{y}=\mathcal{L}_{z}=(x y z) ; \mathcal{R}_{x}=$ $\mathcal{R}_{y}=\mathcal{R}_{z}=e$, so $r$ is non-degenerate, but $r$ is not 2-cancellative, since $x x=$ $y x$ is an equality in $S$. Note that $r(a, b) \neq(a, b), \forall a, b \in X$, so $\mathcal{F}(X, r)=\emptyset$. Moreover, in the group $G(X, r)$ all generators are equal: $x=y=z$.

(b) 19. Let $X=\{x, y, z\}$, and let $r$ be the map

$$
\begin{aligned}
& (x, y) \longrightarrow^{r}(x, z) \longrightarrow^{r}(y, z) \longrightarrow^{r}(y, y) \longrightarrow^{r}(x, y), \\
& (x, x) \longrightarrow^{r}(z, z) \longrightarrow^{r}(y, x) \longrightarrow^{r}(z, y) \longrightarrow^{r}(x, x), \quad r(z, x)=(z, x) .
\end{aligned}
$$

Clearly, $r$ is non-degenerate, $r$ is not 2-cancellative, conditions $\mathbf{1 1}$ and $\mathbf{r} \mathbf{1}$ are satisfied, but $(X, r)$ is not a braided set. Here $\mathcal{F}(X, r)=\{(z, x)\}$.

Lemma 3.6. Suppose $(X, r)$ is a non-degenerate quadratic set (possibly infinite). Then

(1) If $X$ has finite order, $|X|=n$, then

$$
0 \leq|\mathcal{F}(X, r)| \leq|X|=n .
$$

(2) If $(X, r)$ is square-free, then $\mathcal{F}(X, r)=\Delta_{2}$, the diagonal of $X^{2}$. In particular, if $X$ has finite order, then $|\mathcal{F}(X, r)|=|X|=n$.

(3) Suppose $(X, r)$ is non-degenerate and 2-cancellative. Then the following conditions hold.

(a) For every $y \in X$ there exists a unique $x \in X$ such that $r(x, y)=(x, y)$. In other words there exist a bijective map $t: X \longrightarrow X$ such that $r(t(y), y)=(t(y), y)$, for every $y \in X$.

(b) For every $x \in X$ there exists a unique $y \in X$ such that $r(x, y)=(x, y)$.

(c) If $X$ is finite, $X=\left\{x_{1}, \cdots, x_{n}\right\}$, then

$$
\begin{aligned}
& \mathcal{F}=\mathcal{F}(X, r)=\left\{x y \in X^{2} \mid r(x, y)=(x, y)\right\}=\left\{x_{1} y_{1}, \cdots, x_{n} y_{n}\right\}, \\
& \text { where } y_{i} \in X, \text { is the unique element with } r\left(x_{i}, y_{i}\right)=\left(x_{i}, y_{i}\right), 1 \leq i \leq n .
\end{aligned}
$$
In particular, $|\mathcal{F}|=|X|=n$.

Proof. (1). The equality $r(x, y)=\left({ }^{x} y, x^{y}\right)$ implies

$$
(x, y) \in \mathcal{F} \text { if and only if } x=\left({ }^{x} y \text {, and } x^{y}=y .\right.
$$

It follows from the non-degeneracy that for each $y \in X$ there exists unique $x \in X$, such that $x^{y}=y$. In general, it is possible that ${ }^{x} y \neq x$, and in this case $r(x, y)=$ $\left({ }^{x} y, y\right) \neq(x, y)$. This implies (3.3).

(2). Suppose $(X, r)$ is square-free, then, by definition $\Delta_{2} \subseteq \mathcal{F}(X, r)$. Assume $x y \in \mathcal{F}(X, r)$. Then ${ }^{x} y=x={ }^{x} x$, hence by the non-degeneracy, $y=x$. This gives $\mathcal{F}(X, r) \subseteq \Delta_{2}$, and therefore $\mathcal{F}(X, r)=\Delta_{2}$. In particular, if $X$ is finite, then there is an equality $|\mathcal{F}(X, r)|=|X|$

(3). Assume $(X, r)$ is 2-cancellative and nondegenerate. Suppose $y \in X$, then, by the nondegeneracy there exists unique $x \in X$ such that $x^{y}=y$. Consider the equality $r(x, y)=\left({ }^{x} y, x^{y}\right)=\left({ }^{x} y, y\right)$ then by the 2-cancellation law, one has ${ }^{x} y=x$, hence $r(x, y)=(x, y)$, as desired. Assume now that $r(z, y)=(z, y)$ for some 
$z \in X$. Then $r(z, y)=\left({ }^{z} y, z^{y}\right)=(z, y)$ implies $z^{y}=y=x^{y}$, which by the nondegeneracy gives $z=x$. This proves part (3.i). Part (3.ii) is analogous. Part (3.iii) is straightforward from (3.i) and (3.ii).

Corollary 3.7. A non-degenerate quadratic set is square-free if and only if for every pair $x, y \in X$ one has

$$
r(x, y)=(x, y) \Longleftrightarrow x=y .
$$

Remark 3.8. (1) Suppose, $(X, r)$ is a 2-cancelative nondegenerate quadratic set of finite order $|X|=n$. One can apply the theory of (noncommutative) Gröbner bases. We enumerate $X$, as $X=\left\{x_{1}<x_{2}<\cdots<x_{n}\right\}$ and consider the degreelexicographic ordering $\leq$ on $\langle X\rangle$. Let $\mathcal{O}_{j}, 1 \leq j \leq q$, be the set of all nontrivial $r$-orbits. Each $r$-orbit $\mathcal{O}_{j}, 1 \leq j \leq q$, has length $l_{j}=\left|\mathcal{O}_{j}\right| \geq 2$ and contains unique monomial $z_{j} t_{j} \in \mathcal{O}_{j}$, which is minimal (in $\mathcal{O}_{j}$ ) with respect to the ordering $<$ on $\langle X\rangle$. Then the set of defining relations determined by $\mathcal{O}_{j}$, namely $\{x y-r(x y) \mid x y \in$ $\left.\mathcal{O}_{j}\right\}$, reduces to exactly $l_{j}-1$ relations with explicit pairwise distinct highest terms

$$
x y-z_{j} t_{j}=0, x, y \in X, x y \in \mathcal{O}_{j}, x y>z_{j} t_{j} .
$$

The set of reduced relations $\mathbf{R}(r)$ is defined as

$$
\begin{aligned}
& \mathbf{R}(r)=\left\{x y-z_{j} t_{j} \mid x y \in \mathcal{O}_{j}, x y>z_{j} t_{j}, 1 \leq j \leq q\right\}, \\
& |\mathbf{R}(r)|=s=\sum_{1 \leq j \leq q}\left(l_{j}-1\right)=\left(\sum_{1 \leq j \leq q} l_{j}\right)-q \geq q .
\end{aligned}
$$

There is an equality of sets $\Re_{0}(r)=\mathbf{R}(r)$ if and only if $r$ is involutive. Note that the two sets $\Re_{0}(r)$ and $\mathbf{R}(r)$ generate the same two-sided ideal $I$ of $\mathbf{k}\langle X\rangle$. Hence the algebra $A=A(\mathbf{k}, X, r) \cong \mathbf{k}\langle X\rangle /\left(\Re_{0}(r)\right)$ has a finite presentation as $A=\mathbf{k}\langle X\rangle /(\mathbf{R}(r))$. The set of reduced relations $\mathbf{R}(r)$ is exactly the quadratic part of the (minimal) reduced Gröbner basis of $I$, denoted $\mathbf{G R}(I)$ (with respect to the degree-lexicographic ordering $<$ on $\langle X\rangle$ ). The set $\mathbf{R}(r)$ is linearly independent, so $\operatorname{dim} I_{2}=|\mathbf{R}(r)|=s$. In general, $\mathbf{R} \subset \mathbf{G R}(I)$, and the reduced Gröbner basis GR may be infinite. It follows from the theory of Gröbner bases that the set $\mathcal{N}$ of all monomials of length 2 which are normal modulo $I$ (with respect to the degree-lexicographic ordering $<$ on $\langle X\rangle)$ projects to a basis of $A_{2}$. For every integer $m \geq 2$ denote by $\mathcal{N}_{m}$ the set of all monomials in $X^{m}$ which are normal modulo $I=(\mathbf{R}(r))$. Then

$$
\begin{aligned}
\operatorname{dim}_{\mathbf{k}} A_{m} & =\left|\mathcal{N}_{m}\right|=\left|S_{m}\right| \\
& =\text { the number of all disjoint } \mathcal{D}_{m}(r) \text {-orbits in } X^{m} .
\end{aligned}
$$

(2) The Koszul dual algebra $A^{!}$has a presentation $A^{!}=\mathbf{k}\left\langle\xi_{i}, \cdots \xi_{n}\right\rangle /\left(\mathbf{R}^{\perp}\right)$, where $\mathbf{R}^{\perp}$ consists of $s+n$ relations and splits into two disjoint sets

$$
\begin{aligned}
& \mathbf{R}^{\perp}=\mathbf{R}_{0}^{\perp} \cup \mathbf{R}_{1}^{\perp}, \quad \text { where } \\
& \mathbf{R}_{0}^{\perp}=\left\{\xi_{j} \xi_{i}+\xi_{i^{\prime}} \xi_{j^{\prime}} \mid x_{j} x_{i}-x_{i^{\prime}} x_{j^{\prime}} \in \mathbf{R}\right\},\left|\mathbf{R}_{0}^{\perp}\right|=s \\
& \mathbf{R}_{1}^{\perp}=\left\{\xi_{j} \xi_{i} \mid\left(x_{j} x_{i}\right) \in \mathcal{F}(X, r)\right\},\left|\mathbf{R}_{1}^{\perp}\right|=n .
\end{aligned}
$$

There are equalities

$$
\operatorname{dim} A_{2}=n^{2}-s, \quad \operatorname{dim} A_{2}^{!}=n^{2}-s-n .
$$

Suppose, now that $(X, r)$ is a non-degenerate square-free quadratic set (we do not assume 2-cancellativity). Then $\mathcal{F}(X, r)=\Delta_{2}$, and $|\mathcal{F}(X, r)|=n$. Moreover,

$$
\mathbf{R}_{1}^{\perp}=\left\{\xi_{i} \xi_{i} \mid 1 \leq i \leq n\right\} .
$$


Proposition 3.9. Let $(X, r)$ be a non-degenerate quadratic set of finite order $|X|=n \geq 3$, and let $A=A(\boldsymbol{k}, X, r)$ be its associated quadratic $\boldsymbol{k}$-algebra, naturally graded by length. Suppose that $X^{2}$ contains exactly $q$ nontrivial r-orbits, $\mathcal{O}_{1}, \cdots, \mathcal{O}_{q},\left|\mathcal{O}_{j}\right|=l_{j} \geq 2,1 \leq j \leq q$.

(1) If $(X, r)$ is 2-cancellative then the following conditions hold.

(1.a) $X^{2}$ has exactly $n$ one-element $r$-orbits, that is $|\mathcal{F}(X, r)|=n$.

(1.b) The following inequalities hold:

$$
n-1 \leq q \leq\left(\begin{array}{l}
n \\
2
\end{array}\right), \quad \text { and } \quad 2 n-1 \leq \operatorname{dim}_{k} A_{2}=n+q \leq\left(\begin{array}{c}
n+1 \\
2
\end{array}\right),
$$

where the upper bounds for $q$ and for $\operatorname{dim}_{k} A_{2}$ are exact, for all $n \geq 3$, and the lower bounds are exact, whenever $n=p>2$ is a prime number.

(2) Suppose $|\mathcal{F}(X, r)|=n$. Then the following conditions are equivalent

(i) the map $r$ is involutive;

(ii) $\operatorname{dim}_{k} A_{2}=\left(\begin{array}{c}n+1 \\ 2\end{array}\right)$;

(iii) $q=\left(\begin{array}{c}n \\ 2\end{array}\right)$;

(iv) $\operatorname{dim} I_{2}=|\Re(r)|=\left(\begin{array}{l}n \\ 2\end{array}\right)$;

(v) $\operatorname{dim}_{k} A_{2}^{!}=\left(\begin{array}{l}n \\ 2\end{array}\right)$.

Each of these conditions implies that $(X, r)$ is 2-cancellative.

(3) For every integer $m \geq 2, \operatorname{dim}_{k} A_{m}=\left|S_{m}\right|=$ the number of all disjoint $D_{m}$-orbits in $X^{m}$.

Proof. (11). Suppose $(X, r)$ is 2-cancellative. Part (1.a) follows from Lemma 3.6

(1.b). The action of the cyclic group $\langle r\rangle$ on $X^{2}$ splits $X^{2}$ into disjoint $r$-orbits $\mathcal{O}$. We shall analyze the possible number of orbits and their lengths. It is clear that the map $r$ is involutive iff every nontrivial orbit $\mathcal{O}(x y)$ has precisely two elements.

Let $X=\left\{x_{1}, \cdots, x_{n}\right\}$ be an arbitrary enumeration on $X$. By Lemma $3.6 X^{2}$ contains exactly $n$ elements fixed under $r(\mathcal{F}(X, r)$ is described in (3.4) $)$ so there are exactly $n$ one-element $r$-orbits, $\mathcal{O}\left(x_{i} y_{i}\right)=\left\{x_{i} y_{i}\right\}, 1 \leq i \leq n$.

By assumption the complement $X^{2} \backslash \mathcal{F}(X, r)$ splits into $q$ disjoint orbits:

$$
X^{2} \backslash \mathcal{F}(X, r)=\bigcup_{1 \leq j \leq p} \mathcal{O}_{j}, \quad \text { where } \quad\left|\mathcal{O}_{j}\right|=l_{j} \geq 2 .
$$

Then

$$
\left|X^{2} \backslash \mathcal{F}(X, r)\right|=n^{2}-n=\sum_{1 \leq j \leq q} l_{j} .
$$

By the 2-cancellativity of $r$, a nontrivial orbit $\mathcal{O}$ does not contain distinct monomials of the shape $x u, x v, u \neq v$, or $x u, y u, x \neq y$, hence

$$
2 \leq\left|\mathcal{O}_{j}\right|=l_{j} \leq n, \quad \forall 1 \leq j \leq q .
$$

Therefore

$$
2 q \leq \sum_{1 \leq j \leq q} l_{j}=n^{2}-n \leq n q .
$$

But $2 q \leq n^{2}-n$ is equivalent with $q \leq n(n-1) / 2=\left(\begin{array}{l}n \\ 2\end{array}\right)$, moreover $n^{2}-n \leq n q$ implies $n-1 \leq q$. This proves the left-hand side inequalities in 3.7

Recall that $a, b \in \mathcal{O}_{j}$ if and only if $a=b$ in the algebra $A$, or equivalently in the monoid $S$. We argue with the number of distinct words of length 2 in $S$ which is 
the same as the number of all orbits

$$
\left|S_{2}\right|=n+q
$$

One has $A_{2}=\operatorname{Span}_{\mathbf{k}} S_{2}$, and since every set of pairwise distinct words in $S_{2}$ is linearly independent, we yield

$$
2 n-1 \leq \operatorname{dim}_{\mathbf{k}} A_{2}=\left|S_{2}\right|=n+q \leq n+\left(\begin{array}{l}
n \\
2
\end{array}\right)=\left(\begin{array}{c}
n+1 \\
2
\end{array}\right),
$$

which proves the right-hand side inequalities in 3.7 (One may also use the theory of Gröbner basis for a detailed proof, see Remark 3.8. We shall discuss the exactness of the bounds after the proof of part (2).

(2) The equality $\operatorname{dim}_{\mathbf{k}} A_{2}=n+q$ implies the equivalence of (ii) and (iii). The equivalence of (ii) and (v) follows from (3.6).

Note that each $w \in X^{2} \backslash \mathcal{F}$ belongs to a nontrivial orbit $\mathcal{O}(w)$, one has $\left|X^{2} \backslash \mathcal{F}\right|=$ $n(n-1)$. It is clear that $q=\left(\begin{array}{l}n \\ 2\end{array}\right)$ if and only if each nontrivial orbit has exactly two elements, which is equivalent to $r^{2}=1$, this (i) and (iii) are equivalent. One has

$$
\mathbf{k}\langle X\rangle_{2}=I_{2} \oplus A_{2}, \quad \operatorname{dim}(\mathbf{k}\langle X\rangle)_{2}=\operatorname{dim} I_{2}+\operatorname{dim} A_{2},
$$

So

$$
n^{2}-\operatorname{dim} A_{2}=\operatorname{dim} I_{2}=\sum_{1 \leq j \leq q}\left(l_{j}-1\right)=\left(\sum_{1 \leq j \leq q} l_{j}\right)-q
$$

and $\operatorname{dim} I_{2}=\left(\begin{array}{l}n \\ 2\end{array}\right)$ if and only if $\operatorname{dim} A_{2}=\left(\begin{array}{c}n+1 \\ 2\end{array}\right)$, which gives the equivalence of (ii) and (iv).

It follows from [19, Corollary 2.13. that every nondegenerate involutive quadratic set $(X, r)$ is 2-cancellative. Therefore each of the equivalent conditions (i) through (v) implies " $(X, r)$ is 2-cancellative".

Part (2) implies that the upper bounds in (3.7) are exact. If $n=p>2$ is a prime number, then by Corollary 6.18 every square-free self distributive solution, $(X, r)$ corresponding to a dihedral quandle of prime order $|X|=p>2$ satisfies what we call "the minimality condition" : $\operatorname{dim}_{\mathbf{k}} A_{2}=2 n-1$, which is equivalent to $q=n-1$, see Definition 6.1. This proves the exactness of the lower bound, whenever $n=p>2$ is a prime number.

(3). The distinct elements of the monoid $S=S(X, r)$, form a $\mathbf{k}$ - basis of the monoid algebra $\mathbf{k} S \simeq A(\mathbf{k}, X, r)$. In particular $\operatorname{dim} A_{m}$ equals the number of distinct monomials of length $m$ in $S$ which is exactly the number of $\mathcal{D}_{m}(r)$-orbits in $X^{m}$, see Remark 3.8 .

Corollary 3.10. Let $(X, r)$ be a non-degenerate quadratic set of finite order $|X|=$ $n \geq 3$, suppose $|\mathcal{F}(X, r)|=n$. Then the map $r$ is involutive if and only if $\operatorname{dim}_{k} A_{2}=$ $\left(\begin{array}{c}n+1 \\ 2\end{array}\right)$. In this case $(X, r)$ is 2-cancellative.

Corollary 3.11. Let $(X, r)$ be a square-free non-degenerate quadratic set of finite order $|X|=n \geq 3$. Then the map $r$ is involutive if and only if $\operatorname{dim}_{k} A_{2}=\left(\begin{array}{c}n+1 \\ 2\end{array}\right)$. In this case $(X, r)$ is 2-cancellative.

Proposition 3.12. In notation as above. Suppose $(X, r)$ is a non-degenerate quadratic set of finite order $|X|=n$, and $|\mathcal{F}(X, r)|=n$. Let $A=A(\boldsymbol{k}, X, r)$ be its associated quadratic algebra, $A=\boldsymbol{k}\langle X\rangle /(\boldsymbol{R}(r))$.

(1) If $A$ is Koszul, then $r^{2}=i d$ and $|\boldsymbol{R}(r)|=\left(\begin{array}{c}n \\ 2\end{array}\right)$. 
(2) In particular, if there exists an enumeration of $X$, such that the set of quadratic relations $\boldsymbol{R}(r)$ is a Gröbner basis, or equivalently, $A$ is a $P B W$ algebra, then $(X, r)$ is involutive.

(3) In each of the cases: (a) $(X, r)$ is 2-cancellative, or (b) $(X, r)$ is square-free, there is an equality $|\mathcal{F}(X, r)|=n$ so the statement holds.

Proof. (1) Denote $s:=|\mathbf{R}(r)|$. Suppose $A$ is Koszul, so its dual algebra $A^{!}$is also Koszul and their Hilbert series satisfy (3.1). By (3.6) one has

$$
\begin{aligned}
& H_{A}(z)=1+n z+\left(n^{2}-s\right) z^{2}+\left(\operatorname{dim} A_{3}\right) z^{3}+\cdots \\
& H_{A^{!}}(-z)=1-n z+\left(n^{2}-s-n\right) z^{2}-\left(\operatorname{dim} A_{3}^{!}\right) z^{3}+\cdots .
\end{aligned}
$$

We replace these in (3.1) and compute the coefficient for $z^{2}$ to yield:

$$
\left(n^{2}-s-n\right) z^{2}-n^{2} z^{2}+\left(n^{2}-s\right) z^{2}=0,
$$

which implies

$$
|\mathbf{R}(r)|=s=\left(\begin{array}{l}
n \\
2
\end{array}\right) .
$$

Each word $x y \in\left(X^{2} \backslash \mathcal{F}(X, r)\right)$ belongs to a nontrivial $r$-orbit, so $x y$ occurs once in a relation in $\mathbf{R}(r)$. One has $\left|X^{2} \backslash \mathcal{F}(X, r)\right|=n^{2}-n=2\left(\begin{array}{c}n \\ 2\end{array}\right)$. This together with (3.11) implies that each nontrivial $r$-orbit $\mathcal{O}$ in $X^{2}$ has length $|\mathcal{O}|=2$, and therefore $(X, r)$ is involutive. Clearly, in this case $\Re(r)=\mathbf{R}(r)$.

(2) Assume now that there exists an enumeration of $X$, such that the set of quadratic relations $\mathbf{R}(r)$ is a Gröbner basis. Then $A$ is a PBW algebra (in the sense of Priddy), so $A$ is Koszul, and by part (1), $(X, r)$ is involutive.

Corollary 3.13. Let $(X, r)$ be a nondegenerate quadratic set of finite order $|X|=$ $n$, and let $A=A(\boldsymbol{k}, X, r)=\boldsymbol{k}\langle X\rangle /(\Re(r))$, let $I=(\Re(r))$ be the corresponding ideal in $\boldsymbol{k}\langle X\rangle$. Consider the following conditions

(1) $(X, r)$ is involutive.

(2) $(X, r)$ is 2-cancelative.

(3) The set of fixed points $\mathcal{F}(X, r)$ has cardinality $n$.

(4) The number $q$ of nontrivial r-orbits in $X^{2}$ is $q=\left(\begin{array}{l}n \\ 2\end{array}\right)$.

(5) $\operatorname{dim} A_{2}=\left(\begin{array}{c}n+1 \\ 2\end{array}\right)$.

(6) $\operatorname{dim} I_{2}=\left(\begin{array}{l}n \\ 2\end{array}\right)$.

(7) The algebra A has exactly $\left(\begin{array}{l}n \\ 2\end{array}\right)$ defining relations, $|\Re(r)|=\left(\begin{array}{l}n \\ 2\end{array}\right)$.

(8) $\operatorname{dim} A_{2}^{!}=\left(\begin{array}{l}n \\ 2\end{array}\right)$.

(9) The algebra $A$ is Koszul.

The following implications are in force.

$$
\begin{aligned}
& \text { (11) } \Rightarrow \text { (2), (3), (41), (5), (6), (8). } \\
& \text { (2) } \Rightarrow \text { (3) and } n-1 \leq q \leq\left(\begin{array}{l}
n \\
2
\end{array}\right), \quad 2 n-1 \leq \operatorname{dim} A_{2} \leq\left(\begin{array}{c}
n=1 \\
2
\end{array}\right) \text {. } \\
& \text { Assume (2). Then } \\
& \text { (11) } \Leftrightarrow \text { (4) } \Leftrightarrow \text { (5) } \Leftrightarrow \text { (6) } \Leftrightarrow \text { (7) } \Leftrightarrow \text { (8) (9) } \Rightarrow \text { (11). }
\end{aligned}
$$

Lemma 3.14. Suppose $X=\left\{x_{1}, x_{2}, x_{2}\right\}$, and $(X, r)$ is a non-degenerate, 2-cancellative, and square-free quadratic set. 
Then (up to isomorphism) there are exactly three non-isomorphic quadratic algebras $A^{(i)}$ corresponding to quadratic sets $\left(X, r_{i}\right), i=0,1,2$ which satisfy the hypothesis. These are

$$
\begin{aligned}
& A^{(0)}=\boldsymbol{k}\left\langle X: x_{3} x_{2}-x_{2} x_{3}, x_{3} x_{1}-x_{1} x_{3}, x_{2} x_{1}-x_{1} x_{2}\right\rangle \\
& A^{(1)}=\boldsymbol{k}\left\langle X: x_{3} x_{2}-x_{1} x_{3}, x_{3} x_{1}-x_{2} x_{3}, x_{2} x_{1}-x_{1} x_{2}\right\rangle \\
& A^{(2)}=\boldsymbol{k}\left\langle X: x_{3} x_{2}-x_{2} x_{1}, x_{3} x_{1}-x_{1} x_{3}, x_{2} x_{3}-x_{1} x_{2}\right\rangle .
\end{aligned}
$$

The algebras $A^{(0)}$ and $A^{(1)}$ are PBW algebras with GKdim $A=3$ (in fact these are binomial skew-polynomial rings). The quadratic set $\left(X, r_{0}\right)$ is the trivial solution of the YBE. $\left(X, r_{1}\right)$ is the unique (up to isomorphism) non-trivial square-free solution of $Y B E$ of order $|X|=3$. The algebra $A^{(2)}$ is not a PBW algebra with respect to any reordering of $X$, and $\left(X, r_{3}\right)$ is not a solution of the $Y B E$.

Question 3.15. Let $(X, r)$ be a 2-cancellative nondegenerate quadratic set of finite order $|X|=n$. Suppose $A=A(\boldsymbol{k}, X, r)$ is PBW (We know that this implies that $\left.r^{2}=1\right)$. (i). Is it true that if $(X, r)$ is square-free then $A$ has polynomial growth? An equivalent question is: (ii) Is it true that if $(X, r)$ is square-free then $A$ has finite global dimension?

This is so for $|X|=3$, see Lemma 3.14.

An affirmative answer would imply that $(X, r)$ is a solution of $Y B E$, and $A$ satisfies all conditions (1) through (8) in Theorem 3.16. We do not know a counterexample.

In [16] we study the close relation between square-free nondegenerate symmetric sets $(X, r)$ and a class of Artin-Schelter regular algebras. Our result Theorem 2 , investigates quantum binomial quadratic sets $(X, r)$ (that is square-free nondegenerate involutive quadratic set) in terms of various algebraic, homological, and numerical properties of the algebra $A(\mathbf{k}, X, r)$. We have proven that each of these properties of $A$ is equivalent to " $(X, r)$ is a solution of YBE". Our new Theorem 3.16 is a similar but stronger result. We "weaken" the hypothesis, assuming only that $(X, r)$ is a square-free nondegenerate quadratic set (we do not assume involutiveness of $r$ ) and give a list of similar algebraic and homological properties of $A$ each of which is equivalent to " $(X, r)$ is an involutive solution of YBE (that is a symmetric set).

Theorem 3.16. In notation as above. Let $(X, r)$ be a square-free non-degenerate quadratic set of finite order $|X|=n$. Let $A$ be its associated quadratic algebra, $A=\boldsymbol{k}\langle X\rangle /(\boldsymbol{R}(r))$. The following conditions are equivalent.

(1) The Hilbert series of $A$ is

$$
H_{A}(z)=\frac{1}{(1-z)^{n}}
$$

(2) $A$ is a $P B W$ algebra with a set of $P B W$ generators $X=\left\{x_{1}, x_{2}, \cdots, x_{n}\right\}$ and with polynomial growth.

(3) $A$ is a $P B W$ algebra, with a set of $P B W$ generators $X=\left\{x_{1}, x_{2}, \cdots, x_{n}\right\}$ and with finite global dimension, $\operatorname{gl} \operatorname{dim} A<\infty$.

(4) $A$ is a PBW Artin-Schelter regular algebra.

(5) There exists an enumeration of $X, X=\left\{x_{1} \cdots x_{n}\right\}$, such that the set

$$
\mathcal{N}=\left\{x_{1}^{\alpha_{1}} x_{2}^{\alpha_{2}} \cdots x_{n}^{\alpha_{n}} \mid \alpha_{i} \geq 0 \text { for } 1 \leq i \leq n\right\}
$$

is a $\boldsymbol{k}$-basis of $A$. 
$(6)$

$$
\begin{gathered}
\operatorname{dim}_{k} A_{2}=\left(\begin{array}{c}
n+1 \\
2
\end{array}\right), \quad \text { and } \quad \operatorname{dim}_{k} A_{3}=\left(\begin{array}{c}
n+2 \\
3
\end{array}\right) . \\
\operatorname{dim}_{k} A_{2}^{!}=\left(\begin{array}{c}
n \\
2
\end{array}\right), \quad \text { and } \quad \operatorname{dim}_{k} A_{3}^{!}=\left(\begin{array}{c}
n \\
3
\end{array}\right) .
\end{gathered}
$$

(8) $A$ is a binomial skew polynomial ring in the sense of [12].

(9) $(X, r)$ is a square-free symmetric set, that is an involutive solution of $Y B E$.

In this case $A$ is a Noetherian domain. Moreover,

$$
\operatorname{GKdim} A=n=\operatorname{gl} \operatorname{dim} A, \quad \operatorname{dim}_{k} A_{m}=\left(\begin{array}{c}
n+m-1 \\
m
\end{array}\right), m \geq 2 .
$$

Proof. We shall prove that each of conditions (1) through (9) implies that $(X, r)$ is involutive.

Note first that the hypothesis of the theorem implies $|\mathcal{F}(X, r)|=n$. Then by Proposition 3.9 part (2), the map $r$ is involutive iff $\operatorname{dim}_{\mathbf{k}} A_{2}=\left(\begin{array}{c}n+1 \\ 2\end{array}\right)$.

Each of the conditions (6), (77), and (9) implies straightforwardly that $(X, r)$ is involutive. Each of the conditions (2), (3), (44), (5), (8) gives that $A$ is a PBW algebra, hence, by Proposition $3.12(X, r)$ is involutive.

Assume (11) holds. Then $A$ and the algebra of polynomials $P^{n}=\mathbf{k}\left[x_{1}, \cdots, x_{n}\right]$, have the same Hilbert series, $H_{A}(z)=H_{P^{n}}(z)$, see (3.12). Therefore $A$ and $P^{n}$ have the same Hilbert functions

$$
\operatorname{dim} A_{m}=h_{A}(m)=h_{P^{n}}(m)=\left(\begin{array}{c}
n+m-1 \\
m
\end{array}\right), m \geq 1 .
$$

In particular, $\operatorname{dim} A_{2}=\left(\begin{array}{c}n+1 \\ 2\end{array}\right)$, so $r$ is involutive. Moreover, $\operatorname{dim}_{\mathbf{k}} A_{3}=\left(\begin{array}{c}n+2 \\ 3\end{array}\right)$.

We have shown that each of the conditions (1) through (9) implies that $(X, r)$ is a square-free nondegenerate involutive quadratic set, that is $(X, r)$ is a quantum binomial quadratic set, see Definition 1.1](iv). Now our result [16, Theorem 2, implies straightforwardly the equivalence of conditions (1) through (9), the equalities (3.14) and the fact that $A$ is a Noetherian domain.

The 3-generated PBW algebras from Lemma 3.14 are particular cases of the class of PBW algebras described by Theorem 3.16 .

\section{Square-Free quadratic Sets With CyClic Conditions}

In this section we continue the study of square-free nondegenerate quadratic sets $(X, r)$, the associated algebra $A(\mathbf{k}, X, r)$, and the monoid $S(X, r)$. In a series of works, see [13, 19, 18, 6], we have shown that the combinatorial properties of a solution of YBE, $(X, r)$, are closely related to the algebraic and combinatorial properties of its associated structures. Solutions satisfying some of the conditions defined below are of particular interest.

Definition 4.1. [19, 13] Let $(X, r)$ be a quadratic set.

(1) The following are called cyclic conditions on $(X, r)$.

$$
\begin{aligned}
& \text { cl1 : } \quad{ }^{\left(y^{x}\right)} x={ }^{y} x, \quad \text { for all } x, y \in X ; \quad \operatorname{cr} 1: \quad x^{\left({ }^{x} y\right)}=x^{y}, \quad \text { for all } x, y \in X \text {; } \\
& \text { cl2 : } \left.\quad{ }^{x} y\right) x={ }^{y} x, \quad \text { for all } x, y \in X ; \quad \text { cr2 : } \quad x^{\left(y^{x}\right)}=x^{y}, \quad \text { for all } x, y \in X \text {. }
\end{aligned}
$$


(2) Condition lri on $(X, r)$ is defined as

$$
\text { lri: } \quad\left({ }^{x} y\right)^{x}=y={ }^{x}\left(y^{x}\right), \text { for all } \quad x, y \in X .
$$

In other words lri holds if and only if $(X, r)$ is non-degenerate, and

$$
\mathcal{R}_{x}=\mathcal{L}_{x}^{-1}, \quad \text { and } \quad \mathcal{L}_{x}=\mathcal{R}_{x}^{-1} .
$$

The cyclic conditions were introduced by the author in [11, 12, in the context of binomial skew polynomial algebras and were crucial for the proof that every binomial skew polynomial algebra defines canonically (via its relations) a set-theoretic solution of YBE, see [21]. It is known that every square-free non-degenerate symmetric set $(X, r)$ satisfies the cyclic conditions cc and condition lri, so the map $r$ is uniquely determined by the left action: $r(x, y)=\left(\mathcal{L}_{x}(y), \mathcal{L}_{y}^{-1}(x)\right)$, see [13, 19]. We shall prove that every square-free non-degenerate braided set $(X, r)$ (not necessarily finite, or involutive) satisfies the cyclic conditions cl1 and cr1, see Proposition 4.4 The main result of this section is Theorem 4.7 .

4.1. Combinatorics in square-free quadratic sets with cyclic conditions. We recall the following useful result.

Fact 4.2. [19], Proposition 2.25. Suppose $(X, r)$ is a quadratic set. (1) Any two of the following conditions imply the remaining third condition: $(i)(X, r)$ is involutive; (ii) $(X, r)$ is non-degenerate and cyclic; (iii) $(X, r)$ satisfies lri. (2) In particular, if $(X, r)$ satisfies cl1, and $\boldsymbol{c r} \mathbf{1}$ then $(X, r)$ is involutive iff condition lri holds.

Sketch of the proof. For convenience of the reader we shall sketch the proof of (2). Assume lri. We shall prove that $r$ is involutive, or equivalently, (2.1) holds. We apply first cr1 and lri, and next cl1 and lri to yield:

$$
{ }^{u} v\left(u^{v}\right)={ }^{u} v\left(u^{u} v\right)=u, \quad\left({ }^{u} v\right)^{u^{v}}=\left(u^{v} v\right)^{u^{v}}=v, \forall u, v \in X .
$$

Conversely, assume that $(X, r)$ is involutive. We shall prove that lri holds Let $u, t \in X$, we have to show ${ }^{t}\left(u^{t}\right)=u$, and $\left({ }^{t} u\right)^{t}=u$. By the nondegeneracy, there exists $v \in X$, such that $t={ }^{u} v$. Then we use cr1, cl1, and (2.1) to yield:

$$
{ }^{t}\left(u^{t}\right)={ }^{u} v\left(u^{u} v\right)={ }^{u} v\left(u^{v}\right)=u, \quad\left({ }^{t} u\right)^{t}=\left({ }^{u} v u\right)^{u} v=\left({ }^{v} u\right)^{u} v=u .
$$

Suppose $(X, r)$ is a finite nondegenerate quadratic set, $S=S(X, r)$. As we discussed in the preliminaries, for every integer $m \geq 2$ the group $\mathcal{D}_{m}(r)={ }_{g r}\left\langle r^{i i+1}, 1 \leq\right.$ $i \leq m-1\rangle$ acts on the left on $X^{m}$. Each element $a \in S$ can be presented as a monomial $a=\zeta_{1} \zeta_{2} \cdots \zeta_{n}, \quad \zeta_{i} \in X$. Two words $a, b \in\langle X\rangle$ are equal in $S$ if they have the same length, say $a, b \in X^{m}$, and belong to the same orbit of $\mathcal{D}_{m}(r)={ }_{g r}\left\langle r^{i i+1}, 1 \leq i \leq m-1\right\rangle$. Clearly, $(X, r)$ is square-free if and only if $\mathcal{D}_{m}(r)$ acts trivially on $\Delta_{m}, m \geq 2$.

Corollary 4.3. Suppose $(X, r)$ is a square-free quadratic set. Let $x, y \in X$, let $m$ be an integer, $m \geq 2$. If $x^{m}=y^{m}$ is an equality in $S$, then $x=y$.

Proposition 4.4. Let $(X, r)$ be a square-free non-degenerate braided set of arbitrary cardinality. Then $(X, r)$ satisfies the cyclic conditions cl1 and cr1. Moreover, $(X, r)$ is involutive iff condition lri is in force.

Proof. Let $a, x \in X$. Consider the "Yang-Baxter" diagram on monomials of length 3 in $X^{3}$. 


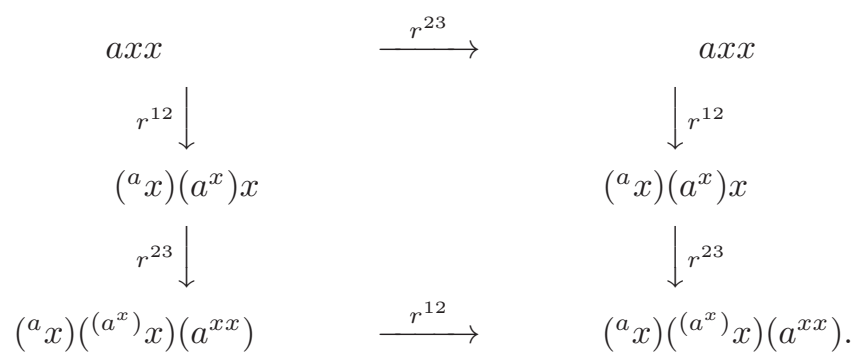

It follows that $r\left({ }^{a} x,{ }^{\left(a^{x}\right)} x\right)=\left({ }^{a} x,{ }^{\left(a^{x}\right)} x\right)$ and therefore, by Corollary $3.7{ }^{\left({ }^{x}\right)} x={ }^{a} x$, which proves cl1.

Similarly, a YB diagram starting with the monomial $x x a$ implies $r\left(x^{\left({ }^{x} a\right)}, x^{a}\right)=$ $\left(x^{\left({ }^{x} a\right)}, x^{a}\right)$, hence $x^{\left({ }^{x} a\right)}=x^{a}$, which proves cr1. Now Fact 4.2 implies straightforwardly that $(X, r)$ is involutive iff lri holds.

The action of the infinite dihedral group $\mathcal{D}$ on $X^{3}$ is of particular importance in this section. Assuming that $(X, r)$ is a nondegenerate square-free quadratic set we shall find some counting formulae and inequalities involving the orders of the $\mathcal{D}$-orbits in $X^{3}$, and their number. As usual, the orbit of a monomial $\omega \in X^{3}$ under the action of $\mathcal{D}$ will be denoted by $\mathcal{O}=\mathcal{O}(\omega)$.

Definition 4.5. We call a $\mathcal{D}$-orbit $\mathcal{O}$ square-free if

$$
\mathcal{O} \bigcap\left(\Delta_{2} \times X \bigcup X \times \Delta_{2}\right)=\emptyset \text {. }
$$

A monomial $\omega \in X^{3}$ is square-free in $S$ if its orbit $\mathcal{O}(\omega)$ is square-free.

Notation 4.6. Denote $\mathbf{E}(\mathcal{O})=\mathcal{O} \cap\left(\left(\Delta_{2} \times X \bigcup X \times \Delta_{2}\right) \backslash \Delta_{3}\right)$.

Theorem 4.7. Suppose $(X, r)$ is a non-degenerate square-free quadratic set, of finite order $|X|=n$.

(1) Let $\mathcal{O}$ be a $\mathcal{D}$-orbit in $X^{3}$. The following implications hold.

$$
\begin{array}{llll}
\text { (i) } & \mathcal{O} \cap \Delta_{3} \neq \emptyset & \Leftrightarrow & |\mathcal{O}|=1 . \\
\text { (ii) } & \boldsymbol{E}(\mathcal{O}) \neq \emptyset & \Longrightarrow & |\mathcal{O}| \geq 3 .
\end{array}
$$

In this case we say that $\mathcal{O}$ is a $\mathcal{D}$-orbit of type (ii).

(iii) $\mathcal{O} \cap\left(\Delta_{2} \times X \cup X \times \Delta_{2}\right)=\emptyset \Longrightarrow|\mathcal{O}| \geq 6$.

Recall that in this case $\mathcal{O}$ is called a square-free orbit, see Definition 4.5 .

(2) The following two conditions are equivalent

(a) $(X, r)$ is involutive and satisfies the cyclic conditions cl1 and cr1;

(b) Every orbit $\mathcal{O}$ of type (ii) contains exactly 3 distinct elements.

Proof. Condition (11) (i) is clear.

(11) (ii). Assume that $\mathbf{E}(\mathcal{O}) \neq \emptyset$. Then $\mathcal{O}$ contains an element of the shape $\omega=x x y$, or $\omega=x y y$, where $x, y \in X, x \neq y$. Without loss of generality we can assume $\omega=x x y \in \mathcal{O}$. We look at a fragment of the "Yang-Baxter" diagram starting with $\omega$ :

$$
\omega=\omega_{1}=x x y \longrightarrow{ }^{23} \omega_{2}=x\left({ }^{x} y\right)\left(x^{y}\right) \longrightarrow{ }^{r^{12}} \omega_{3}=\left(x^{x^{2}} y\right)\left(x^{x} y\right)\left(x^{y}\right) \longrightarrow \cdots .
$$

Note that the first three elements $\omega_{1}, \omega_{2}, \omega_{3}$ are distinct monomials in $X^{3}$. Indeed, $x \neq y$ implies $r(x y) \neq x y$ in $X^{2}$, (see condition (1)), so $\omega_{2} \neq \omega_{1}$. By assumption 
$(X, r)$ is square-free, so ${ }^{x} x=x$, and $y \neq x$, implies ${ }^{x} y \neq x$, by the nondegeneracy. Therefore $r\left(x\left({ }^{x} y\right)\right) \neq x\left({ }^{x} y\right)$, and $\omega_{3} \neq \omega_{2}$. Furthermore, $\omega_{3} \neq \omega_{1}$. Indeed, if we assume $x={ }^{x^{2}} y={ }^{x}\left({ }^{x} y\right)$ then by (2.2) one has ${ }^{x} y=x$, and therefore $y=x$, a contradiction. It follows that $|\mathcal{O}| \geq 3$.

(11) (iii). Suppose $\mathcal{O}=\mathcal{O}(x y z)$ is a square-free $\mathcal{D}$-orbit in $X^{3}$. Consider the set

$$
O_{1}=\left\{v_{i} \mid 1 \leq i \leq 6\right\} \subseteq \mathcal{O}
$$

consisting of the first six elements of the "Yang-Baxter" diagram

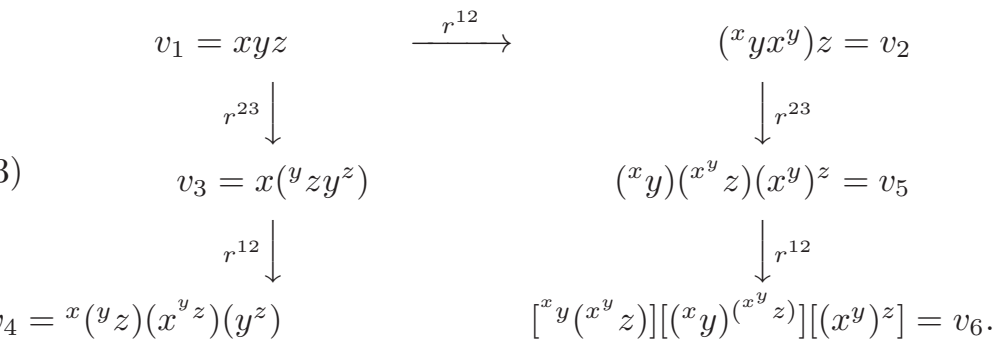

Clearly,

$$
O_{1}=U_{1} \bigcup U_{3} \bigcup U_{5}, \quad \text { where } U_{j}=\left\{v_{j}, v_{j+1}=r^{12}\left(v_{j}\right)\right\}, \quad j=1,3,5 .
$$

We claim that $U_{1}, U_{3}, U_{5}$ are pairwise disjoint sets, and each of them has order 2. Note first that since $v_{j}$ is a square-free monomial, for each $j=1,3,5$, one has $v_{j} \neq r_{12}\left(v_{j}\right)=v_{j+1}$, therefore $\left|U_{j}\right|=2, \quad j=1,3,5$. The monomials in each $U_{j}$ have the same "tail". More precisely, $v_{1}=(x y) z, v_{2}=r(x y) z$, have a "tail" $z$, the tail of $v_{3}$, and $v_{4}$ is $y^{z}$, and the tail of $v_{5}$, and $v_{6}$ is $\left(x^{y}\right)^{z}$. It will be enough to show that the three elements $z, y^{z},\left(x^{y}\right)^{z} \in X$ are pairwise distinct. But $\mathcal{O}(x y z)$ is square-free, so $y \neq z$ and by (2.2) $y^{z} \neq z$. Furthermore $v_{2}=\left({ }^{x} y\right)\left(x^{y}\right) z \in \mathcal{O}(x y z)$, so $x^{y} \neq y$ and $x^{y} \neq z$. Now by the nondegeneracy one has

$$
x^{y} \neq z \Longrightarrow\left(x^{y}\right)^{z} \neq z \quad x^{y} \neq y \Longrightarrow\left(x^{y}\right)^{z} \neq y^{z} .
$$

Therefore the three elements $z, y^{z},\left(x^{y}\right)^{z} \in X$ occurring as tails in $U_{1}, U_{3}, U_{5}$, respectively, are pairwise distinct, so the three sets are pairwise disjoint. This implies $6=\left|O_{1}\right| \leq|\mathcal{O}|$.

(2). (a) $\Rightarrow$ (b). Suppose $(X, r)$ is involutive and satisfies cl1 and cr1. Let $\mathcal{O}$ be an orbit of type (ii). Without loss of generality we may assume $\mathcal{O}=\mathcal{O}(x x y)$. Then (since $r$ is involutive) each arrow in the diagram (4.4) is pointed in both directions, i.e. the arrows have the shape $\longleftrightarrow$, or $\uparrow$.

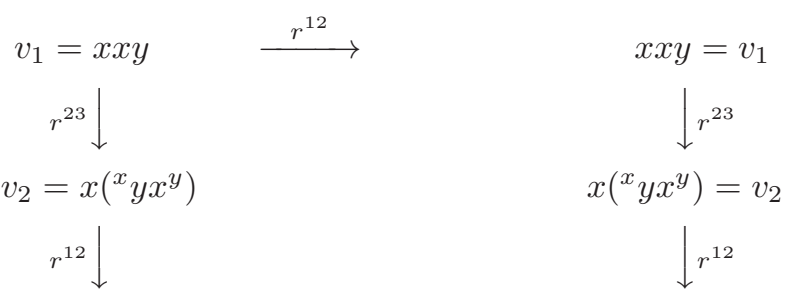

$$
v_{3}=\left({ }^{x}\left({ }^{x} y\right)\right)\left(x^{x} y\right)\left(x^{y}\right)=\left({ }^{x x} y\right) x^{y} x^{y} \quad \stackrel{r^{23}}{\longrightarrow} \quad\left({ }^{x}\left({ }^{x} y\right)\right)\left(x^{x} y\right)\left(x^{y}\right)=\left({ }^{x x} y\right)\left(x^{y}\right)\left(x^{y}\right)=v_{3}
$$

It is clear that the diagram contains all elements of $\mathcal{O}$, hence $|\mathcal{O}|=3$. 
(b) $\Rightarrow$ (a). Suppose every orbit $\mathcal{O}$ of type (ii) contains exactly three elements. The diagram

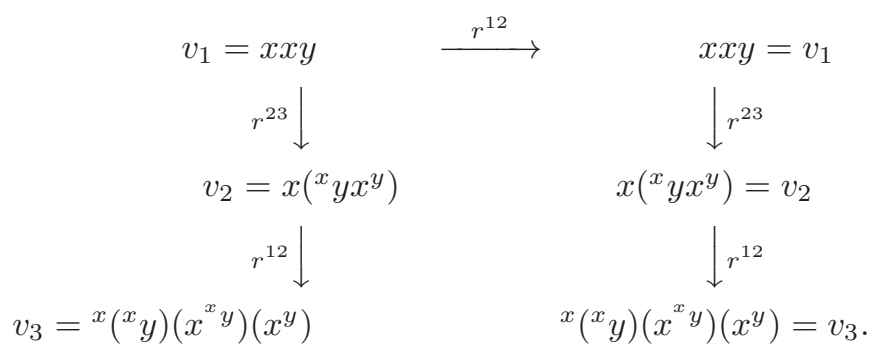

contains three distinct elements of $\mathcal{O}$, and therefore it contains the whole orbit $\mathcal{O}$.

The element $r^{23}\left(v_{3}\right)={ }^{x}\left({ }^{x} y\right) r\left(\left(x^{x} y\right)\left(x^{y}\right)\right) \in \mathcal{O}=\left\{v_{1}, v_{2}, v_{3}\right\}$. It is clear that $r^{23}\left(v_{3}\right) \neq v_{1}$ and $r^{23}\left(v_{3}\right) \neq v_{2}$, so $r^{23}\left(v_{3}\right)=v_{3}$. This implies that $r\left(\left(x^{x} y\right)\left(x^{y}\right)\right)=$ $\left(x^{x} y\right)\left(x^{y}\right)$, hence $\left(x^{x} y\right)\left(x^{y}\right) \in \mathcal{F}(X, r)=\Delta_{2}$. Therefore

$$
x^{x} y=x^{y}, \forall x, y \in X,
$$

that is cr1 holds. An analogous argument proves cl1 (in this case we work with a "YB" diagram with a left top element $\left.v_{1}=x y y\right)$.

Notice that if there exists a pair $(x, y)$ with $r^{2}(x y) \neq x y$ then the orbit $\mathcal{O}(x x y)$ contains (but is not limited to) the following four distinct elements

$$
\begin{aligned}
& v_{1}=x x y, \quad v_{2}=r^{23}\left(v_{1}\right)=x r(x y)=x\left({ }^{x} y x^{y}\right), \\
& v_{3}=r^{12}\left(v_{2}\right)=\left({ }^{x} y\right)\left(x^{x} y\right)\left(x^{y}\right), \quad v_{4}=r^{23}\left(v_{2}\right)=x r^{2}(x y)
\end{aligned}
$$

which contradicts (b). It follows that $r$ is involutive. We have proven (b) $\Rightarrow$ (a).

4.2. More on square-free quadratic sets with cyclic conditions. We end up the section with new results on square-free quadratic sets which will be used to describe the contrast between involutive and noninvolutive solutions of YBE in the next section.

Proposition 4.8. Suppose $(X, r)$ is a finite non-degenerate square-free quadratic set satisfying cl1 and $\mathrm{cr} \mathbf{1}$, let $|X|=n$. Then $(X, r)$ is a symmetric set if and only if $\operatorname{dim}_{k} A_{3}^{!}=\left(\begin{array}{l}n \\ 3\end{array}\right)$.

Proof. By hypothesis cl1 and $\mathbf{c r} \mathbf{1}$ hold. Assume $\operatorname{dim}_{\mathbf{k}} A_{3}^{!}=\left(\begin{array}{l}n \\ 3\end{array}\right)$. We have to show that $(X, r)$ is a symmetric set. We shall prove first that $(X, r)$ is involutive, and therefore it is a quantum binomial set, see Definition 1.1 (iv).

As usual, we study the $\mathcal{D}_{3}$-orbits $\mathcal{O}$. Our assumption implies that $X^{3}$ contains exactly $\left(\begin{array}{l}n \\ 3\end{array}\right)$ square-free orbits, $\mathcal{O}^{(s)}, 1 \leq s \leq\left(\begin{array}{l}n \\ 3\end{array}\right)$. By Theorem 4.7 part (1)iii) the length of each square-free orbit satisfies

$$
\left|\mathcal{O}^{(s)}\right|=l_{s} \geq 6, \quad \forall 1 \leq s \leq\left(\begin{array}{l}
n \\
3
\end{array}\right) .
$$

Denote by $W$ the set of all words $w \in X^{3} \backslash \Delta_{3}$ which vanish in $A_{3}^{!}$. Note first that if $y, b \in X, y \neq b$, the orbit $\mathcal{O}(y y b) \subset W$ contains the three distinct monomials occurring in the following diagram

$$
u=y y b \longrightarrow_{r^{23}} y\left({ }^{y} b y^{b}\right) \longrightarrow_{r^{12}}\left({ }^{y y} b\right)\left(y^{y} b \cdot y^{b}\right)=\left({ }^{y y} b\right)\left(y^{b} \cdot y^{b}\right)
$$


We shall call the word $r^{23}(y y b)=y\left({ }^{y} b y^{b}\right)$ "the transition element for the pair of words $u=y y b, c z z=r^{12} \circ r^{23}(u) \in W^{\prime \prime}$. It is clear that each pair $y, b \in X, y \neq b$ determines uniquely the three elements $y y b, r^{23}(y y b), c z z=r^{12} \circ r^{23}(y y b) \in W$.

Note that if $(y, b) \neq(t, c)$ then the transition elements $y\left({ }^{y} b y^{b}\right) \neq t\left({ }^{t} c t^{c}\right)$. Indeed, the inequality is straightforward if $t \neq y$. If $t=y, b \neq c$, then by the nondegeneracy, one has ${ }^{y} b \neq{ }^{y} c={ }^{t} c$. So $W$ contains $n(n-1)$ disjoint triples $y y b, r^{23}(y y b), r^{12} \circ$ $r^{23}(y y b)=c z z$, therefore $|W| \geq 3 n(n-1)$.

Assume that $(X, r)$ is not involutive, we shall prove that $|W|>3 n(n-1)$.

Clearly, there exist a pair $x, a \in X, x \neq a$, such that $r^{2}(x, a) \neq(x, a)$ so the words $x a, r(x a), r^{2}(x a)$ are distinct elements of $X^{2}$. Then the orbit $\mathcal{O}=\mathcal{O}(x x a)$ contains at least the set $\mathbf{O}$ of 4 distinct monomials given below:

$$
\mathbf{O}=\left\{\begin{array}{l}
v_{1}=x x a, v_{2}=r^{23}(x x a)=x\left({ }^{x} a x^{a}\right), \\
v_{3}=r^{12} \circ r^{23}\left(v_{1}\right)=\left({ }^{x x} a\right)\left(x^{a} x^{a}\right), \\
\left.v_{4}=\left(r^{23}\right)^{2}\left(v_{1}\right)=x r^{2}(x a)\right\} .
\end{array}\right.
$$

Moreover, the set $\mathbf{O}$ contains the word $v_{4}=x r^{2}(x a)$ which is square-free, but is not a transition element for any triple $y y b, r^{23}(y y b), r^{12} \circ r^{23}(y y b)=c z z$. This implies that

$$
|W|>3 n(n-1) .
$$

The set $X^{3}$ splits into the following disjoint subsets

$$
X^{3}=\Delta_{3} \bigcup W \bigcup\left(\bigcup_{1 \leq s \leq\left(\begin{array}{c}
n \\
3
\end{array}\right)} \mathcal{O}^{(s)}\right) .
$$

This, together with (4.6) and (4.8) imply

$$
n^{3}=\left|X^{3}\right|=\left|\Delta_{3}\right|+|W|+\sum_{1 \leq s \leq\left(\begin{array}{c}
n \\
3
\end{array}\right)}\left|\mathcal{O}^{(s)}\right|>n+3 n(n-1)+6\left(\begin{array}{l}
n \\
3
\end{array}\right)=n^{3},
$$

which gives a contradiction. It follows that $r$ is involutive, hence $(X, r)$ is a quantum binomial set. Now our result [16, Theorem 2, implies that $(X, r)$ is a solution of YBE, therefore, it is a symmetric set. The inverse implication follows again form [16, Theorem 2.

Lemma 4.9. Let $(X, r)$ be a finite square-free non-degenerate quadratic set, $|X|=$ $n$, and let $S=S(X, r)$. Suppose $(X, r)$ satisfies the cyclic conditions cl1 and $\boldsymbol{c r} \mathbf{1}$. The following conditions are equivalent:

(1) $(X, r)$ is involutive.

(2) $S$ satisfies the following conditions:

$$
\begin{aligned}
& a x x=\text { byy holds in } S, a, b, x, y \in X \Longrightarrow a=b, x=y \\
& x x c=y y d \text { holds in } S, c, d, x, y \in X \Longrightarrow c=d, x=y .
\end{aligned}
$$

$$
\begin{aligned}
& \text { byy } \in \mathcal{O}(a x x) \quad a, b, x, y \in X \Longrightarrow a=b, x=y \\
& y y d \in \mathcal{O}(x x c) \quad c, d, x, y \in X \Longrightarrow c=d, x=y .
\end{aligned}
$$

Proof. The equivalence (2) $\Leftrightarrow(3)$ is clear.

(11) $\Rightarrow$ (2). Suppose $(X, r)$ is involutive. Theorem 4.7 implies that for each $a \neq x$ the orbit $\mathcal{O}=\mathcal{O}(a x x)$ is of type (ii) and $\mathcal{O} \bigcap\left(X \times \Delta_{2}\right)=\{a x x\}$, in other words 
there is no element of the shape byy $\neq a x x$ such that byy $\in \mathcal{O}$ which gives the first implication in (4.10). Analogous argument gives the second implication in (4.10).

(2) $\Rightarrow$ (11) Conversely, assume that conditions (4.10) are in force. We have to show that $r$ is involutive. By Fact 4.2 it will be enough to prove that $(X, r)$ satisfies condition lri, that is

$$
\left({ }^{t} x\right)^{t}=x \text { and } \quad{ }^{t}\left(x^{t}\right)=x, \forall x, t \in X .
$$

Let $a, x \in X$. We consider the elements in the $\mathcal{D}$-orbit $\mathcal{O}(a x x)$ in $X^{3}$ and deduce the following equalities of elements in $S=S(X, r)$ :

$$
\begin{aligned}
& a . x x=\left({ }^{a} x\right)\left(a^{x}\right) x \\
& =\left({ }^{a} x\right)\left({ }^{a^{x}} x\right) a^{x x}=\left({ }^{a} x\right)\left({ }^{a} x\right)\left(a^{x x}\right) \\
& =\left({ }^{a} x\right)\left({ }^{a} x\left(a^{x x}\right)\right)\left(\left({ }^{a} x\right)^{a^{x x}}\right) \\
& =\left({ }^{\left({ }^{a} x\right.}\left({ }^{a} x\left(a^{x x}\right)\right)\right)\left(\left({ }^{a} x\right)^{\left({ }^{a}{ }^{x}\left(a^{x x}\right)\right)}\left(\left({ }^{a} x\right)^{\left(a^{x x}\right)}\right)\right. \\
& =b\left(\left({ }^{a} x\right)^{a^{x x}}\right)\left(\left({ }^{a} x\right)^{a^{x x}}\right) \quad \text { where } \quad b=\left({ }^{a} x\right)\left(\left({ }^{a} x\right)\left(a^{x x}\right)\right) \\
& \text { =byy, where } y=\left[\left({ }^{a} x\right)\right]^{\left(a^{x x}\right)} \text {. }
\end{aligned}
$$

We have obtained that for $a \neq x$ the following equalities holds in $S$

$$
a x x=b y y, \text { where } y=\left[\left({ }^{a} x\right)\right]^{\left(a^{x x}\right)} .
$$

Now the first condition in 4.10) implies

$$
y=\left[\left({ }^{a} x\right)\right]^{\left(a^{x x}\right)}=x,
$$

and

$$
\begin{aligned}
& { }^{a} x={ }^{a^{x}} x={ }^{\left(a^{x}\right)^{x}} x={ }^{\left(a^{(x x)}\right)} x \\
& \left.x=\left[\left({ }^{a} x\right)\right]\right]^{\left(a^{x x}\right)}=\left[{ }^{\left({ }^{(x x)}\right)} x\right]^{\left(a^{x x}\right)}=\left({ }^{t} x\right)^{t}, \quad \text { where } t=a^{(x x)} .
\end{aligned}
$$

Suppose $t, x \in X$. By the nondegeneracy there exists $a_{1} \in X$, such that $t=a_{1}^{x}$, and, similarly, there exists $a \in X$ with $a^{x}=a_{1}$, hence $t=a^{x x}$, for some $a \in X$. Then (4.14) implies $\left({ }^{t} x\right)^{t}=x$. The second equality ${ }^{t}\left(x^{t}\right)=x$ in (4.11) is proven by an analogous argument. Therefore $(X, r)$ satisfies condition lri.

\section{Square-Free Braided Sets And the Contrast Between the INVOLUTIVE AND NONINVOLUTIVE CASES}

Braided monoids were introduced and studied [19]. For convenience of the reader we recall basis definitions and results in Section 8. Recall that if $(X, r)$ is a braided set then its monoid $S(X, r)$ is a graded braided M3 monoid, we denote it by $\left(S, r_{S}\right)$, see Definitions 8.1 and 8.2 , in particular, $S$ satisfies condition ML2. More details can be found in in Section 8 .

Notation 5.1. Let $(X, r)$ be a nondegenerate quadratic set. Let $a, x \in X$ and let $m$ be a positive integer. We shall use the following notation

$$
{ }^{\left(x^{m}\right)} a:=\left(\mathcal{L}_{x}^{m}\right)(a) \quad a^{\left(x^{m}\right)}:=\left(\mathcal{R}_{x}^{m}\right)(a) .
$$

This formulae agree with the natural left and right actions of $S$ upon itself.

Remark 5.2. Suppose $(X, r)$ is a quadratic set with $\mathbf{c l 1}$ and $\mathbf{c r} \mathbf{1}$. Then the following equalities hold in $X$ :

$$
{ }^{a^{\left(x^{m}\right)}} x={ }^{a} x, \quad x^{\left(x^{m}\right)} a=x^{a}, \quad \text { for all } a, x \in X \text { and all positive integers } m .
$$

The formulae (5.1) are easy to prove using induction on $m$. 
Proposition 5.3. Let $(X, r)$ be a square-free non-degenerate quadratic set satisfying the cyclic conditions cl1 and cr1. and let $S=S(X, r)$. Then the following conditions hold.

(1) For every pair $a, x \in X$ and every positive integer $m$ the following equalities hold in $S$.

$$
\text { a. }\left(x^{m}\right)=\left(\left({ }^{a} x\right)^{m}\right) \cdot\left(a^{\left(x^{m}\right)}\right), \quad\left(x^{m}\right) \cdot a=\left({ }^{\left(x^{m}\right)} a\right)\left(\left(x^{a}\right)^{m}\right),
$$

(2) Assume that $(X, r)$ is a braided set. Then the following are equalities in the braided monoid $S(X, r)$.

$$
{ }^{a}\left(x^{m}\right)=\left({ }^{a} x\right)^{m}, \quad\left(x^{m}\right)^{a}=\left(x^{a}\right)^{m}, \text { for all } a, x \in X \text { and all } m \in \mathbb{N}
$$

(3) Suppose that $X$ is finite, and let $p$ be the least common multiple of the orders of all actions, $\mathcal{L}_{x}$ and $\mathcal{R}_{x}, x \in X$, so ${ }^{\left(x^{p}\right)} a=a=a^{\left(x^{p}\right)}, \forall a, x \in X$. Then the following equalities hold in $S$ :

$$
\begin{gathered}
a \cdot\left(x^{p}\right)=a \cdot\left(\left({ }^{a} x\right)^{a}\right)^{p}, \quad\left(x^{p}\right) \cdot a=\left({ }^{a}\left(x^{a}\right)\right)^{p} \cdot a, \forall a, x \in X . \\
\left(x^{p}\right)\left(y^{p}\right)=\left(y^{p}\right)\left(x^{p}\right), \forall x, y \in X .
\end{gathered}
$$

Proof. (11). We shall use induction on $m$ to prove the first equality in (5.2). Clearly, for $m=1$, one has $a x={ }^{a} x \cdot x^{a}$. Assume $a \cdot\left(x^{k}\right)=\left(\left({ }^{a} x\right)^{k}\right) \cdot\left(a^{\left(x^{k}\right)}\right), \forall 1 \leq k \leq m$, and all $a, x \in X$. Let $a, x \in X$. Then

$$
\begin{aligned}
a \cdot\left(x^{m+1}\right) & =\left(a \cdot x^{m}\right) x=\left(\left({ }^{a} x\right)^{m}\right) \cdot\left[\left(a^{\left(x^{m}\right)}\right) x\right]=\left({ }^{a} x\right)^{m} \cdot\left({ }^{\left(a^{\left(x^{m}\right)}\right.} x\right) \cdot\left(a^{\left(x^{m}\right)}\right)^{x} \\
& \left.=\left({ }^{a} x\right)^{m+1}\right)\left(a^{\left(x^{m+1}\right)}\right),
\end{aligned}
$$

as claimed. This verifies the first equality in (5.2). Analogous argument verifies the second equality in (5.2)

(2). Assume that $(X, r)$ is a braided set. Then $(X, r)$ satisfies cl1 and $\mathbf{c r} \mathbf{1}$, see Proposition 4.4. We shall prove (5.3) using induction on $m$. The base for induction is clear. Assume the formula is true for all $k \leq m-1$, where $m \geq 2$. We use the inductive assumption, ML2 and (5.1) to yield

$$
{ }^{a}\left(x^{m}\right)={ }^{a}\left(\left(x^{m-1}\right) \cdot x\right)=\left({ }^{a}\left(x^{m-1}\right)\right) \cdot\left(\left({\left(x^{\left(x^{m-1}\right)}\right)}^{2}\right)=\left({ }^{a} x\right)^{m-1}\right)\left({ }^{a} x\right)=\left({ }^{a} x\right)^{m} .
$$

This proves the first equality in (5.3). Analogous argument verifies the second equality in (5.3).

(3). Assume now that $X$ is a finite set and $p$ is the least common multiple of the orders of all actions. We use successively M3, (5.3) and M3 again to yield

$$
\left.a \cdot\left(x^{p}\right)={ }^{a}\left(x^{p}\right) \cdot\left(a^{\left(x^{p}\right)}\right)=\left({ }^{a} x\right)^{p} \cdot a=\left(\left({ }^{a} x\right)^{p}\right) a\right) \cdot\left(\left({ }^{a} x\right)^{p}\right)^{a}=a \cdot\left(\left({ }^{a} x\right)^{a}\right)^{p} .
$$

This gives the first equality in (5.4). Analogous argument proves the second equality in (5.4). The equality (5.5) is straightforward.

Proposition 5.4. Let $(X, r)$ be a finite square-free non-degenerate braided set, $S=S(X, r)=\left(S, r_{S}\right)$ be the associated braided monoid, and let $A=A(\boldsymbol{k}, X, r)$. Let $p$ be the least common multiple of the orders of all actions, $\mathcal{L}_{x}$ and $\mathcal{R}_{x}, x \in X$. The following conditions are equivalent

(1) The equality $a x^{p}=a y^{p}$ in $S$, for $a, x, y \in X$, implies $x=y$;

(2) The equality $\left(x^{p}\right) a=\left(y^{p}\right) a$ in $S, a, x, y \in X$, implies $x=y$.

(3) The monoid $S$ is cancellative.

(4) The quadratic algebra $A$ has Gelfand-Kirillov dimension $G K \operatorname{dim} A=n$. 
(5) The solution $(X, r)$ is involutive, that is $(X, r)$ is a symmetric set.

In this case $S(X, r)$ is embedded in the braided group $G(X, r)=\left(G, r_{G}\right)$. Moreover, both $\left(S, r_{S}\right)$ and $\left(G, r_{G}\right)$ are also (non-degenerate) involutive solutions.

Proof. The implication (3) $\Longrightarrow$ (10), (2), is clear.

(11) $\Longrightarrow$ (2). Assume $\left(x^{p}\right) a=\left(y^{p}\right) a$, where $a, x, y \in X$. Then we use (5.2) to obtain

$$
\left(x^{p}\right) a=a\left(x^{a}\right)^{p}, \quad\left(y^{p}\right) a=a\left(y^{a}\right)^{p} .
$$

It follows that $a\left(x^{a}\right)^{p}=a\left(y^{a}\right)^{p}$, so by our assumption (1), $x^{a}=y^{a}$. Hence, by the non-degeneracy, $x=y$. The implication (2) $\Longrightarrow$ (1) is proven analogously.

(11) $\Longrightarrow$ (5). Suppose condition (1) holds (hence (2) is also in force). Proposition (5.3) implies the following equalities in $S$ :

$$
a \cdot\left(x^{p}\right)=a \cdot\left(\left({ }^{a} x\right)^{a}\right)^{p}, \quad\left(x^{p}\right) \cdot a=\left({ }^{a}\left(x^{a}\right)\right)^{p} \cdot a, \forall a, x \in X .
$$

It follows from (10) that

$$
x=\left(\left({ }^{a} x\right)^{a}\right) ; \quad x=\left({ }^{a}\left(x^{a}\right)\right), \forall a, x \in X,
$$

therefore the braided set $(X, r)$ satisfies condition lri. By Fact $4.2(2),(X, r)$ is involutive, so $(X, r)$ is a non-degenerate symmetric set.

$(5) \Longrightarrow$ (3). It is known, see [10, that if $(X, r)$ is a non-degenerate symmetric set then its monoid $S(X, r)$ is embedded in the group $G(X, r)$, and therefore $S$ is left and right cancellative.

The implication (5) $\Longrightarrow$ (4) is known, see [16, Theorem 2, or [21.

(4) $\Longrightarrow$ (11). Suppose $A$ has Gelfand-Kirillov dimension $G K \operatorname{dim} A=n$. Assume, on the contrary, that condition (11) is not satisfied. Then there exist three elements $a, x, y \in X, a \neq x, y$, such that

$$
a x^{p}=a y^{p}, x \neq y .
$$

This implies that $a x^{M p}=a y^{M p}$, for all positive integers $M$, hence GKdim $A<n$, a contradiction.

The following result shows the close relations between various algebraic and combinatorial properties of a finite square-free solution $(X, r)$, the YB-algebra $A=$ $A(\mathbf{k}, X, r)$ and its braided monoid, $S=S(X, r)$. Each of these conditions describes the contrast between a square-free symmetric set and a square-free noninvolutive braided set.

Theorem 5.5. Let $(X, r)$ be a square-free non-degenerate braided set of order $|X|=$ $n$. Let $S=S(X, r)$ be its braided monoid, let $A=A(\boldsymbol{k}, X, r)$ be its graded $\boldsymbol{k}$ - algebra over a field $\boldsymbol{k}$, and let $A^{!}$be the Koszul dual algebra of $A$. The following conditions are equivalent:

(1) The solution $(X, r)$ is involutive, that is $(X, r)$ is a symmetric set.

(2) $(X, r)$ satisfies lri.

(3) The Hilbert series of $A$ is $H_{A}(z)=\frac{1}{(1-z)^{n}}$.

(4) The quadratic algebra $A$ is Koszul.

(5) There exists an enumeration of $X$, such that the set of quadratic relations $\boldsymbol{R}(r)$ is a Gröbner basis basis, that is $A$ is a $P B W$ algebra.

(6) $A$ is a binomial skew polynomial ring, (in the sense of [12]) with respect to an enumeration of $X$. 
(7) $\operatorname{dim}_{k} A_{2}=\left(\begin{array}{c}n+1 \\ 2\end{array}\right)$.

(8) $\operatorname{dim}_{k} A_{3}=\left(\begin{array}{c}n+2 \\ 3\end{array}\right)$.

(9) $\operatorname{dim}_{k} A_{3}^{!}=\left(\begin{array}{l}n \\ 3\end{array}\right)$.

(10) The algebra $A$ has Gelfand-Kirillov dimension GKdim $A=n$. (Meaning that the integer-valued function $i \mapsto \operatorname{dim}_{k} A_{i}$ is bounded by a polynomial in $i$ of degree $n$ ).

(11) If $a x^{p}=a y^{p}$ holds in $S, a, x, y \in X$ then $x=y$, where $p$ is the least common multiple of the orders of all actions $\mathcal{L}_{x}$ and $\mathcal{R}_{x}, x \in X$.

(12) The monoid $S$ satisfies conditions 4.10)

(13) The monoid $S$ is cancellative.

(14) $A$ is a domain.

Each of these conditions implies that $A$ is an Artin-Schelter regular algebra of global dimension $n$.

Proof. Note first that $(X, r)$ satisfies cl1, and $\mathbf{c r 1}$, by Proposition 4.4. Moreover, $|\mathcal{F}(X, r)|=n$. It is known that a finite square-free nondegenerate symmetric set $(X, r)$ satisfies all conditions (2) through (14) in the theorem, so (1) implies all conditions (2) through (14). These implications have been published in various works of the author, but you can find them all in [16], Theorem 2. The remaining implications with references to the corresponding results follow.
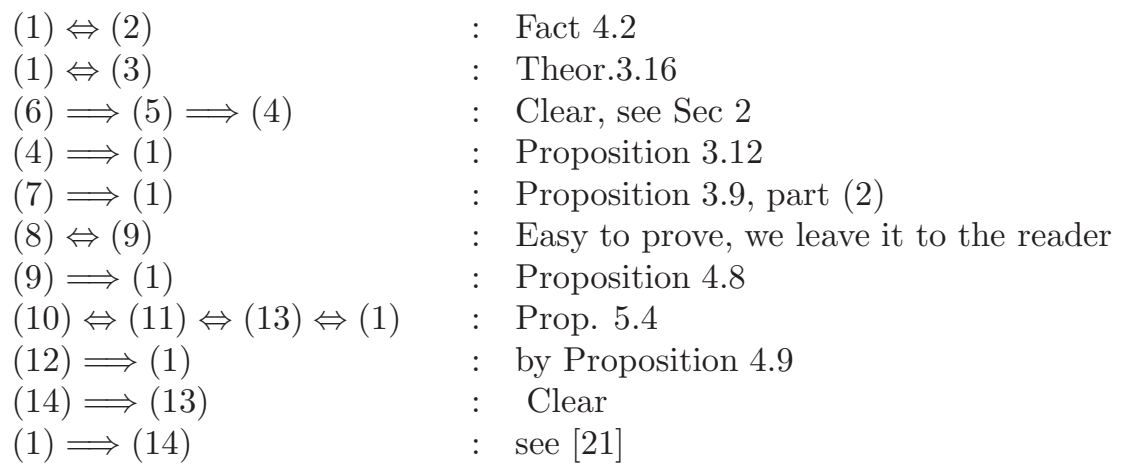

Corollary 5.6. (Characterization of noninvolutive square-free braided sets) Under the hypothesis of Theorem 5.5. Let $(X, r)$ be a square-free non-degenerate braided set of order $|X|=n, S(X, r), A=A(\boldsymbol{k}, X, r)$, and $A^{!}$in usual notation. Suppose $r^{2} \neq i d_{X \times X}$. Then the following conditions hold.

(1) $(X, r)$ does not satisfy condition lri.

(2) The algebra $A$ is not Koszul.

(3) The set of quadratic relations $\boldsymbol{R}(r)$ is not a Gröbner basis with respect to any enumeration of $X$.

(4) $A$ is a not a binomial skew polynomial ring, with respect to any enumeration of $X$.

(5) $2 n-1 \leq \operatorname{dim}_{k} A_{2} \leq\left(\begin{array}{c}n+1 \\ 2\end{array}\right)-1$.

(6) $\operatorname{dim}_{k} A_{3}<\left(\begin{array}{c}n+2 \\ 3\end{array}\right)$.

(7) $0 \leq \operatorname{dim}_{k} A_{3}^{!}<\left(\begin{array}{c}n \\ 3\end{array}\right)$, and $A_{3}^{!}=0$, whenever $\operatorname{dim}_{k} A_{2}=2 n-1$.

(8) $G K \operatorname{dim} A<n$. 
(9) Suppose $p$ is the least common multiple of the orders of all actions, $\mathcal{L}_{x}$ and $\mathcal{R}_{x}, x \in X$. Then there exist pairwise distinct elements $a, x, y \in X$, such that $a x^{p}=a y^{p}$ holds in $S$.

(10) There exist $x, y \in X$, such that $x \neq y$, and $x^{p}=y^{p}$ holds in the group $G(X, r)$.

(11) There exist $a, b, x, y \in X$, such that $x \neq y, x \neq a, y \neq b$, and the equality axx = byy holds in $S$.

(12) The monoid $S=S(X, r)$ is not cancellative. In particular, $S(X, r)$ is not embedded in the group $G(X, r)$.

(13) The algebra $A$ is not a domain.

Remark 5.7. The lower bound $2|X|-1 \leq \operatorname{dim}_{\mathbf{k}} A_{2}$ is exact, whenever $|X|=p, p$ is a prime number. More precisely, a Dihedral quandle $(X, \triangleright)$ of prime order $|X|=p$ satisfies condition $\mathbf{M}$, see Lemma 6.16.

Question 5.8. (1) For which integers $n$ this lower bound is attainable, that is there exists a braided set $(X, r),|X|=n$ satisfying the minimality condintion $M$ ? (2) Classify the square-free solutions $(X, r)$ satisfying the minimality condition $M$.

Note that every 2-cancelative square-free solution $(X, r)$ with minimality condition $\mathbf{M}$ is indecomposable, see Lemma 6.6. However, being indecomposable is not sufficient- there exists indecomposable solutions with $\operatorname{dim}_{\mathbf{k}} A_{2}>2|X|-1$, see Example 6.21

Remark 5.9. We have shown that if $(X, r)$ is a finite non-degenerate square-free braded set, where $r$ is not involutive, then the monoid $S=S(X, r)$ is not cancellative (even if $(X, r)$ is 2-cancellative). This gives a negative answer to Open Question 3.24 in [19: "Is it true that if $(X, r)$ is a 2-cancellative braided set, then the associated monoid $S(X, r)$ is cancellative?"

Conjecture 5.10. Let $(X, r)$ be an arbitrary finite non-degenerate braided set with 2-cancellation. Then the monoid $S(X, r)$ is cancellative if and only if $r$ is involutive.

6. Square-Free BRAided Sets $(X, r)$ SATisfying the Minimality Condition

6.1. Square-free 2-cancellative quadratic sets $(X, r)$ with minimality condition.

Definition 6.1. We say that a finite quadratic set $(X, r)$ satisfies the minimality condition $\mathbf{M}$ if

$$
\mathbf{M}: \quad \operatorname{dim}_{\mathbf{k}} A_{2}=2|X|-1 \text {. }
$$

Example 6.2. Every square-free self distributive solution, $(X, r)$ (see Definition 6.8), corresponding to a dihedral quandle of prime order $|X|=p>2$ satisfies the minimality condition $\mathbf{M}$, see Corollary 6.18

Let $(X, r)$ be a square-free non-degenerate quadratic set set of order $|X|=n$. Assume that $(X, r)$ is 2-cancellative. Let $S=S(X, r)$ be its graded monoid, let $A=A(\mathbf{k}, X, r)$ be its graded k- algebra over a field $\mathbf{k}$, and let $A^{!}$be the Koszul dual algebra. Consider the action of $\mathcal{D}_{3}(r)=\operatorname{gr}_{\operatorname{gr}}\left\langle r^{12}, r^{23}\right\rangle$ on $X^{3}$. The following useful remarks are straightforward.

Remark 6.3. In assumption as above the following are equivalent: 
(1) Each $\mathcal{D}_{3}(r)$-orbit in $X^{3}$ contains a word of the type $x x y, x, y \in X$ (and $z t t, z, t \in X)$.

(2) $A_{3}^{!}=0$.

Remark 6.4. Let $(X, r)$ be a square-free non-degenerate quadratic set of order $|X|=n$, assume that $(X, r)$ is 2 -cancellative. Suppose $\mathcal{O}$ is a nontrivial $r$-orbit in $X^{2}$ of order $|\mathcal{O}|=n$. Then (i) for every $x \in X$ there exists $y \in X$ such that $x y \in \mathcal{O}$; (ii) for every $y \in X$ there exists $x \in X$, such that $x y \in \mathcal{O}$.

Proposition 6.5. Let $(X, r)$ be a square-free non-degenerate quadratic set of order $|X|=n, X=\left\{x_{1}, \cdots, x_{n}\right\}, S=S(X, r), A=A(\boldsymbol{k}, X, r)$, and $A^{!}$in usual notation. Assume that $(X, r)$ is 2-cancellative. Let $\mathcal{O}_{i}, 1 \leq i \leq q$ be the set of all nontrivial $r$ - orbits in $X^{2}$ (these are exactly the square-free r-orbits in $X^{2}$ ).

(1) The following three conditions are equivalent.

(1a) $(X, r)$ satisfies the minimality condition $\boldsymbol{M}$, 6.1);

(1b) Each non-trivial orbit $\mathcal{O}_{i}$ has order $\left|\mathcal{O}_{i}\right|=n$.

(1c) The algebra $A$ has a finite presentation $A \cong \boldsymbol{k}\langle X\rangle /\left(\boldsymbol{R}_{0}\right)$, where $\boldsymbol{R}_{0}$ is a set of exactly $(n-1)^{2}$ quadratic square-free binomial relations:

$$
\boldsymbol{R}_{0}=\left\{x_{i n} y_{i n}-x_{1} x_{i}, x_{i n-1} y_{i n-1}-x_{1} x_{i}, \cdots, x_{i 2} y_{i 2}-x_{1} x_{i} \mid 2 \leq i \leq n\right\},
$$

where $x_{i j} \neq y_{i j}, 1 \leq i, j \leq n$, and the following two conditions hold for every $2 \leq i \leq n$ :

(c1) $x_{i n} y_{i n}>x_{i n-1} y_{i n-1}>\cdots>x_{i 2} y_{i 2}>x_{i 1} y_{i 1}=x_{1} x_{i}$;

(c2) there are equalities of sets

$$
\left\{x_{i j} \mid 2 \leq j \leq n\right\}=X \backslash\left\{x_{1}\right\}, \quad\left\{y_{i j} \mid 2 \leq j \leq n\right\}=X \backslash\left\{x_{i}\right\} .
$$

In this case, after a possible re-enumeration of the orbits, one has

$\mathcal{O}_{i}=\mathcal{O}\left(x_{1} x_{i}\right)=\left\{x_{1} x_{i}:=x_{i 1} y_{i 1}<x_{i 2} y_{i 2}<\cdots<x_{i n-1} y_{i n-1}<x_{i n} y_{i n}\right\}, 2 \leq i \leq n$.

(2) Moreover, each of conditions (1a), (1b), (1c) implies that

(i) $A_{3}^{!}=0$. In particular, $X^{3}$ does not contain square-free $\mathcal{D}_{3}(r)$-orbits.

(ii) $\operatorname{GKdim} A \leq 2$.

Proof. Recall first that for arbitrary quadratic set $(X, r)$ the number of distinct words of length 2 in $S$ is exactly the number of $\mathcal{D}_{2}(r)$-orbits in $X^{2}$, see Remark 2.9 So one has

$$
\operatorname{dim} A_{2}=\left|S_{2}\right|=\sharp\left(\mathcal{D}_{2} \text {-orbits in } X^{2}\right) .
$$

(1). (1a) $\Longleftrightarrow(1 \mathrm{~b})$. Suppose the minimality condition (6.1) holds. Then $X^{2}$ splits into exactly $2 n-1$ orbits, more precisely there are $n$ one element orbits, these are the elements of the diagonal $\Delta_{2}$ and $n-1$ square-free orbit $\mathcal{O}_{i}, 1 \leq i \leq n-1$. Due to 2-cancellativity one has $\left|\mathcal{O}_{i}\right| \leq n$. At the same time the following hold:

$$
n^{2}-n=\left|\bigcup_{1 \leq i \leq n-1} \mathcal{O}_{i}\right|=\sum_{1 \leq i \leq n-1}\left|\mathcal{O}_{i}\right| \leq n(n-1) .
$$

Therefore $\left|\mathcal{O}_{i}\right|=n$, for all $1 \leq i \leq n-1$.

Conversely, suppose each nontrivial orbit $\mathcal{O}$ has length $n$, and let $q$ be the number of square-free orbits $\mathcal{O}$. There are exactly $(n-1) n$ square-free words in $X^{2}$, each is contained in some $\mathcal{O}$, so $n(n-1)=n q$, and therefore $q=n-1$. Thus the total number of disjoint orbits in $X^{2}$ is $n+(n-1)=2 n-1$. It follows that $\left|S_{2}\right|=2 n-1$, and $\operatorname{dim}_{\mathbf{k}} A_{2}=\left|S_{2}\right|=2 n-1$, which is exactly the minimality condition $\mathbf{M}$. 
$(1 \mathrm{~b}) \Longrightarrow(1 \mathrm{c})$. Suppose each non-trivial $\mathcal{D}_{2}(r)$ - orbit $\mathcal{O}_{i}$ in $X^{2}$ has order $\left|\mathcal{O}_{i}\right|=$ $n, 1 \leq i \leq n-1$. It follows from Remark 6.4 that for each $1 \leq i \leq n-1$ there exist unique $x \in X$, such that $x_{1} x \in \mathcal{O}_{i}$. We re-enumerate the orbits (if necessary), so that $x_{1} x_{i} \in \mathcal{O}_{i}$. Let $1 \leq i \leq n-1$. We order lexicographically the $n$ (distinct) words in $\mathcal{O}_{i}$ :

$$
\mathcal{O}_{i}=\left\{x_{i n} y_{i n}>x_{i n-1} y_{i n-1}>\cdots x_{i 2} y_{i 2}>x_{i 1} y_{i 1}=: x_{1} x_{i}\right\} .
$$

Each two monomials in $\mathcal{O}_{i}$, considered as elements of $S$, are equal. This information is encoded by the set $\mathbf{R}_{i}$ of exactly $n-1$ "reduced" relations determined by $\mathcal{O}_{i}$ :

$$
\mathbf{R}_{i}: \quad x_{i n} y_{i n}=x_{1} x_{i} ; x_{i n-1} y_{i n-1}=x_{1} x_{i} ; \cdots, x_{i 2} y_{i 2}=x_{1} x_{i} .
$$

As discussed in Section 3 the set of defining relations $\Re(r)$ is equivalent to the set of reduced relations

$$
\mathbf{R}=\bigcup_{i} \mathbf{R}_{i}
$$

and the corresponding "algebra-type" relations are exactly the $n(n-1)$ reduced relations $\mathbf{R}_{0}$ given in (6.2). It follows from the properties of the orbits $\mathcal{O}_{i}$ that the relations in $\mathbf{R}_{0}$ satisfy all additional conditions in part (1c).

$(1 \mathrm{c}) \Longrightarrow(1 \mathrm{~b})$. The relations $\mathbf{R}_{0}$ imply the $n-1$ sets of relations $\mathbf{R}_{i}, 1 \leq i \leq$ $n-1$. Note that the properties of the relations given in part (3) imply that $(X, r)$ is 2-cancellative. It is clear that a relation $a=b \in \mathbf{R}_{i}$ implies that $a, b$ belong to the same orbit $\mathcal{O}$ in $X^{2}$. We denote this orbit by $\mathcal{O}_{i}$ (we have $x_{1} x_{i} \in \mathcal{O}_{i}$ ). One can also read off from the properties of $\mathbf{R}_{i}$ that $\mathcal{O}_{i}$ has exactly $n$-elemnts. Note that the sets $\Re_{0}(r)$ and $\mathbf{R}_{0}$ generate the same two-sided ideal $I$ of $\mathbf{k}\langle I\rangle$, so we get $A \cong \mathbf{k}\langle X\rangle /\left(\mathbf{R}_{0}\right)$. It follows from the theory of Gröbner bases that the ideal $I$ has unique reduced Gröbner basis basis $\mathbf{G R}(\mathrm{I})$ (w.r.t $\leq$ ). Moreover, $\mathbf{R}_{0}$ is a proper subset of $\mathbf{G R}(I)$, (we assume $n \geq 3$ ) and all additional elements of $\mathbf{G R}(I) \backslash \mathbf{R}_{0}$ are homogeneous polynomials of degree $\geq 3$. Therefore, the set of normal monomials of length 2:

$$
\mathcal{N}_{2}=\left\{x_{1} x_{2}, x_{1} x_{3}, \cdots, x_{1} x_{n}\right\} \bigcup\left\{x_{i} x_{i} \mid 1 \leq i \leq n\right\}
$$

projects to a $\mathbf{k}$-basis of $A_{2} \cong \mathbf{k} S_{2}$, so this again implies $\operatorname{dim}_{\mathbf{k}} A_{2}=2 n-1$.

(2) Suppose $(X, r)$ satisfies the minimality condition $\mathbf{M}$. It follows from the argument in part (1) that the normal basis $\mathcal{N}$ of $A$ satisfies

$$
\mathcal{N} \subseteq\left\{x_{1}^{\alpha} x_{i}^{\beta} \mid 2 \leq i \leq n, \alpha \geq 0, \beta \geq 0\right\} .
$$

This implies that GKdim $A \leq 2$.

We shall prove that $A^{!}=0$. By Remark 6.3 it will be enough to show that each $\mathcal{D}_{3}(r)$-orbit in $X^{3}$ contains a word of the type $x x y, x, y \in X$ (and $z t t, z, t \in X$ ). Let $a, b, c \in X$. Without loss of generality, we may assume that $a \neq b$, and $b \neq c$. Clearly, $b c \in \mathcal{O}(b c) \subset X^{2}$, and by Remark 6.4 the (square-free) orbit $\mathcal{O}(b c)$ contains an element of the shape $a t, t \in X$, thus $b c=a t$ is an equality in $S_{2}$, so $a b c=a a t$ holds in $S_{3}$. This implies that the $\mathcal{D}_{3}$-orbit $\mathcal{O}(a b c)$ in $X^{3}$ contains the monomial aat, and therefore it is not square-free. It follows then that there are no square-free orbits in $X^{3}$, hence $A^{!}=0$.

Lemma 6.6. Suppose $(X, r)$ is a finite square-free non-degenerate braided set which is 2-cancellative, and satisfies the minimality condition $M$. Let $|X|=n \geq 3$. Then $(X, r)$ is indecomposable. 
Proof. By Proposition 6.5 $(X, r)$ satisfies the minimality conditions iff $X^{2} \backslash \Delta_{2}$, , the set of square-free words of length 2 , splits into $n-1$ disjoint $\mathcal{D}_{2}$-orbits $\mathcal{O}_{i}, 1 \leq$ $i \leq n-1$, each of which contains $n$ distinct monomials. Suppose $X=Y \bigcup Z$ is a decomposition into nonempty disjoint $r$-invariant subsets, say $|Y|=k \geq 2,|Z|=$ $s \geq 1, k+s=n$. Let $\left(Y, r_{Y}\right)$ be the corresponding subsolution. Let $x, y \in Y, x \neq y$, then the $\mathcal{D}_{2}$-orbit $\mathcal{O}(x y)$ in $X^{2}$ is contained entirely in $Y^{2}$, one has $\mathcal{O}(x y)=\mathcal{O}_{i}$, for some $1 \leq i \leq n-1$. But $\left(Y, r_{Y}\right)$ is also square-free and 2-cancelative solution of order $k$, hence $|\mathcal{O}(x y)| \leq k<n$, which, by Proposition 6.5 gives a contradiction.

Note that being indecomposable does not guarantee that a braided set $(X, r)$ satisfies the minimality condition. Example 6.21 gives a square-free braided set $(X, r)$ which is indecomposable (and injective), but does not satisfy the minimality condition $\mathbf{M}$.

Question 6.7. Suppose $(X, r)$ is a square-free 2-cancellative solution. What can be said about $(X, r)$ if its quadratic algebra $A$ satisfies $A_{3}^{!}=0$ ? In particular, is it true that if $A_{3}^{!}=0$ then $(X, r)$ satisfies the minimality condition $\boldsymbol{M}$ ?

\subsection{Some basics on indecomposable injective racks.}

Definition 6.8. We call a quadratic set $(X, r)$ (left) self distributive or shortly SD if it satisfies

$$
\text { SD : } \quad r(x, y)=\left({ }^{x} y, x\right), \forall x, y \in X .
$$

Remark 6.9. Suppose $(X, r)$ is an SD quadratic set.

(1) The following implications are in force

$(X, r)$ is 2-cancellative $\Longrightarrow(X, r)$ is non-degenerate.

$(X, r)$ is 2-cancellative $\Longrightarrow{ }^{x} x=x, \forall x \in X \Longleftrightarrow(X, r)$ is square-free.

(2) $(X, r)$ is involutive iff $(X, r)$ is the trivial solution.

(3) $(X, r)$ is a braided set iff the condition laut holds:

$$
\operatorname{laut}(\mathbf{x}, \mathbf{y}, \mathbf{z}): \quad{ }^{x}\left({ }^{y} z\right)={ }^{x} y\left({ }^{x} z\right), \forall x, y, z \in X .
$$

Self distributive braided sets are closely related to racks. We recall some basics on racks, we follow [1].

Definition 6.10. 1 $A$ rack is a pair $(X, \triangleright)$, where $X$ is a nonempty set, and $\triangleright: X \times X \longrightarrow X$ is a map (a binary operation on $X$ ) such that

(R1) The map $\varphi_{i}: X \longrightarrow X, x \mapsto i \triangleright x$ is bijective for all $i \in X$, and

(R2) $i \triangleright(j \triangleright k)=(i \triangleright j) \triangleright(i \triangleright k)$.

$A$ quandle is a rack which also satisfies $i \triangleright i=i$, for all $i \in X$.

$A$ crossed set is a quandle such that $j \triangleright i=i$, whenever $i \triangleright j=j$.

Remark 6.11. Suppose $(X, r)$ is an SD quadratic set. Define $\triangleright: X \times X \longrightarrow X$ as $x \triangleright y:={ }^{x} y$. It is clear that $(X, \triangleright)$ is a rack iff $(X, r)$ is a non-degenerate braided set. Moreover, $(X, r)$ is a square-free braided set iff $(X, \triangleright)$ is a quandle.

Conversely, every rack $(X, \triangleright)$ defines canonically a non-degenerate SD braided set $(X, r)$, where $r(x, y)=(x \triangleright y, y)$. Moreover $(X, r)$ is square-free iff $(X, \triangleright)$ is a quandle. It follows from Remark 6.9 that every rack $(X, r)$ which is 2 -cancellative is a quandle.

To simplify notation and terminology, " a non-degenerate braided set $(X, r)$ satisfying SD" will be referred to as "a rack" and if in addition $(X, r)$ is square-free 
it will be also referred to as "a quandle". Under this convention we shall keep notation and shall write "xy", instead of " $x \triangleright y$ ". In this case there is an equality of maps:

$$
\varphi_{x}=\mathcal{L}_{x}, \forall x \in X .
$$

The inner group, $\operatorname{Inn}(\mathrm{X})$ of a rack $X$ is the subgroup of $\operatorname{Sym}(X)$ generated by all permutations $\mathcal{L}_{x}, x \in X$. A rack $(X, r)$ is faithful if the map $X \longrightarrow \operatorname{Inn}(\mathrm{X})$, $x \longmapsto \mathcal{L}_{x}$ is injective. In fact, $X$ is indecomposable if and only if $\operatorname{Inn}(\mathrm{X})$ acts transitively on $X$.

Example 6.12. Let $n$ be a positive integer. Over the ring $\mathbb{Z} / n \mathbb{Z}$ of integers $\bmod$ $n$ define $x \triangleright y=2 x-y$. This is a quandle known as the dihedral quandle of order $n$. This ia an Alexander quandle, see for example [30. If we assume that $\mathrm{n}$ is odd, we can identify the elements of this quandle with the conjugacy class of involutions of $D_{n}$, the dihedral group of order $2 n$. Classification of Alexander quandles of prime order $p$ can be found for example in 30.

These are particular cases of affine racks. Let $X$ be an abelian group and let $g$ be an automorphism of $X$. Then $x \triangleright y=(1-g)(x)+g(y)$ is a rack, an affine rack over $X$.

The following results are extracted from $[22]$

Fact 6.13. Suppose $(X, r)$ is a finite $\boldsymbol{S D}$ braided set, and assume that the corresponding rack $(X, \triangleright)$ is indecomposable. Then

(1) $(X, \triangleright)$ is faithful iff it is injective (Lemma 2.10, 22]).

Clearly, in this case the solution $(X, r)$ is also injective.

(2) Suppose $X=\left\{x_{1}, \cdots, x_{n}\right\}$. Then all permutation $\mathcal{L}_{i}, i \in X$ have the same order $m$. Moreover, the equalities $x_{i}^{m}=x_{j}^{m}$ hold in $G_{X}$ for all $, 1 \leq i, j \leq n$.

(3) For all $x \in X$ the permutation $\mathcal{L}_{x}$ has exactly $1+k_{2}$ fixed points, where $k_{2}$ is the number of elements $j \in X$ such that $\mathcal{O}(1, j)$ has 2 elements, see Lemma 2.25.3, 22]).

Remark 6.14. Suppose that $(X, r)$ is an indecomposable quandle of order $|X| \geq 3$, and every nontrivial $r$-orbit $\mathcal{O} \subset X^{2}$ has order $3 \leq|\mathcal{O}| \leq|X|$. Then (i) for every $x \in X$ the permutation $\mathcal{L}_{x}$ has unique fixed point, namely $x$. (ii) Moreover, if $\mathcal{L}_{x}^{2}=\mathrm{id}, \forall x \in X$, then $X$ has an odd order $|X|=2 k+1$. In this case $\mathcal{L}_{x}$ is a product of $k$ disjoint transposition.

\subsection{Quandles with minimality condition $\mathrm{M}$.}

Corollary 6.15. Suppose $(X, r)$ is a 2-cancellative $\boldsymbol{S D}$ braided set of finite order $n=|X| \geq 3$, assume that $\mathcal{L}_{x}^{2}=i d, \forall x \in X$.

If $(X, r)$ satisfies the minimality condition $\boldsymbol{M}$ (hence $X$ is indecomposable), then (i) $X$ has an odd order, $n=2 k+1$, and (ii) each $\mathcal{L}_{x}, x \in X$ is a product of $k$ disjoint transpositions.

The following result is well known to the experts.

Lemma 6.16. If $(X, r)$ is a dihedral quandle of prime order $|X|=p$ then each non-trivial $r$-orbit $\mathcal{O}$ in $X^{2}$ has length exactly $p$.

Proof. Let $x, y \in X, x \neq y$. Then, by definition, $r(x, y)=(2 x-y, x)$. One proves by induction that

$$
r^{k}(x, y)=((k+1) x-k y, k x-(k-1) y)
$$


But all maps $r^{k}$ are bijections and $(k+1) x-k y=x$ if and only if $k=0 \bmod p$, which implies that the $r$-orbit $\mathcal{O}(x, y)$ in $X^{2}$ has size $p$.

Recall that the dihedral quandles, and the Alexander quandles are well known since decades. A classification of Alexander quandles of prime order $p$ can be found for example in [30. The particular proof of Lemma 6.16 was kindly provided to us by Leandro Vendramin.

Definition 6.17. If (X,r) is a dihedral quandle of prime order $|X|=p$, it is called an Alexander quandle. It can be identified with the set of reflections of a regular $p$-gon (elements of the dihedral group $D_{2 p}$ ).

Corollary 6.18. Suppose that $(X, r)$ is a square-free $\boldsymbol{S D}$ solution, corresponding to a dihedral quandle of order $|X|=p>2$, where $p$ is a prime number. Then $(X, r)$ satisfies the minimality condition $\boldsymbol{M}$.

Questions 6.3.1. The following questions refer to finite square-free solutions $(X, r)$ which are 2-cancellative.

(1) Is it true that if a dihedral quandle $(X, r)$ satisfies the minimality condition $\mathbf{M}$ then its order $|X|$ is a prime number?

(2) Suppose $(X, \triangleright)$ is an indecomposable quandle such that the corresponding solution $(X, r)$ satisfies the minimality condition $\mathbf{M}$. Does this imply that the quandle $(X, \triangleright)$ is simple?

(3) Which of the known simple quandles satisfy the minimality condition $\mathbf{M}$ ?

(4) Study general square-free noninvolutive, braided sets $(X, r)$ which are not SD.

(5) Classify the square-free noninvolutive, braided sets $(X, r)$ whose quadratic algebra satisfy GKdim $A(\mathbf{k}, X, r)=1$.

(6) Classify the square-free noninvolutive braided sets of small orders. In particular, classify the square-free noninvolutive, and not SD braided sets $(X, r)$ of small order.

(7) Find examples of indecomposable (not SD) finite square-free solutions;

(8) Find examples of indecomposable (not SD) square-free solutions which satisfy the minimality conditions $\mathbf{M}$.

6.4. Concrete examples of quandles. We have applied our results on squarefree solutions $(X, r)$ to find various examples, as solutions on the following natural problem.

Problem 6.4.1. Given the following data: (a) A set $X$ of odd cardinality $n=2 k+1$; (b) a cyclic permutation $r_{0} \in \operatorname{Sym}\left(X^{2} \backslash \Delta_{2}\right)$ of order $n$

$$
\mathcal{O}: a_{1} b_{1} \longrightarrow_{r_{0}} a_{2} b_{2} \longrightarrow_{r_{0}} \cdots \longrightarrow_{r_{0}} a_{n} b_{n} \longrightarrow_{r_{0}} a_{1} b_{1}
$$

where $a_{i} \neq b_{i}, 1 \leq i \leq n, a_{i} \neq a_{j}, b_{i} \neq b_{j}$, whenever $i \neq j, 1 \leq i, j \leq n$.

Find an extension $r: X \times X \longrightarrow X \times X$ of $r_{0}$ (equivalently, find all maps $\mathcal{L}_{x}, x \in X$ explicitly), so that

(i) $(X, r)$ is a 2-cancellative square-free $\mathbf{S D}$ braided set, with 2-cancellation;

(ii) $\mathcal{L}_{x}^{2}=$ id, $\forall x \in X$.

Analyze the obtained solutions (if any). In particular (a) decide whether this data determine an SD solution with minimality condition $\mathbf{M}$; (b) what kind of quandles can be obtained this way? 
We give next concrete examples. The first illustrates a solution of Problem 6.4.1 on a quadratic set $(X, r)$ of order 5 .

Example 6.19. Suppose $(X, r)$ is a quadratic SD set of order $|X|=5$, so $r(x, y)=$ $\left({ }^{x} y, x\right)$. To simplify notation we set $X=\{1,2,3,4,5\}$, as it is often used for racks. Assume that $\mathcal{L}_{x}^{2}=\mathrm{id}, \forall x \in X$. Suppose $\langle r\rangle$ has an orbit of length 5, say:

$$
\mathcal{O}(12): \quad 54 \longrightarrow_{r} 35 \longrightarrow_{r} 23 \longrightarrow_{r} \mathbf{1 2} \longrightarrow_{r} 41 \longrightarrow_{r} 54
$$

Then the following conditions hold.

(1) The orbit (6.7) determines the maps $\mathcal{L}_{i}, i \in X$ (and $r$ ) uniquely, so that $(X, r)$ is a non-degenerate 2 -cancelative braided set. More precisely, $(X, r)$ is a braided set iff the left actions are:

$$
\begin{array}{lll}
\mathcal{L}_{1}=(24)(35), & \mathcal{L}_{2}=(13)(45), & \mathcal{L}_{3}=(25)(14) \\
\mathcal{L}_{4}=(15)(23), & \mathcal{L}_{5}=(34)(12) .
\end{array}
$$

(2) In this case $X^{2}$ splits into $4 r$-orbits of length 5: $\mathcal{O}(1 i), 2 \leq i \leq 5$, and 5 one-element orbits for the elements of the diagonal $\operatorname{diag}\left(X^{2}\right)$.

(3) Consider the degree-lexicographic ordering $\leq$ on $\langle X\rangle$, induced by the ordering $1<2<3<4<5$ on $X$. The set of defining relations $\Re(r)$ reduces (and is equivalent) to the following set of 16 quadratic relations:

$$
\mathbf{R}=\left\{\begin{array}{llll}
54=12 & 41=12 & 35=12 & 23=12 \\
53=14 & 45=14 & 32=14 & 21=14 \\
52=15 & 43=15 & 31=15 & 24=15 \\
51=13 & 42=13 & 34=13 & 25=13\}
\end{array}\right.
$$

(4) The braided set $(X, r)$ is self distributive and satisfies the minimality condition $\mathrm{M}$. Let $(X, \triangleright)$ be the quandle canonically associated with $(X, r)$. This quandle is isomorphic to the dihedral quandle of order 5 . The solution $(X, r)$ is also injective.

(5) Let $A=A(\mathbf{k}, X, r)=\mathbf{k}\langle X ; \mathbf{R}\rangle \cong \mathbf{k}\langle X\rangle /(I)$ be the associated quadratic algebra graded by length. The ideal $I$ is generated by the set

$$
\mathbf{R}_{0}=\{u-v \mid u=v \in \mathbf{R}\} \subset \mathbf{k}\langle X\rangle .
$$

It is not difficult to find that the reduced Gröbner basis $\mathbf{G B}\left(\mathbf{R}_{0}\right)$ contains 4 additional relations:

$\mathbf{G B}\left(\mathbf{R}_{0}\right)=\mathbf{R}_{0} \bigcup\{155-122,144-122,133-122,1222-1112\}$.

It follows that $A$ is standard finitely presented.

(6) $A$ is left and right Noetherian.

(7) $G K \operatorname{dim} A=1 ; A_{3}^{!}=0$

(8) The monoid $S$ satisfies the relations (6.9) and also the following relations derived from the Gröbner basis

$$
155=122,144=122,133=122,1222=1112 .
$$

In particular $S$ is not 3-cancellative, hence $S$ is not cancellative .

(9) The group $G(X, r)$ satisfies the relations (6.9) which (only in the group case) give rise to the following new relations in $G$ :

$$
55=44=33=22=11 .
$$

We have deduced these relations straightforwardly from the Gröbner basis (without using the theory of racks). Of course, they agree with Fact 6.13 
Example 6.20. Suppose $(X, r)$ is an SD quadratic set of order 9. Assume that $\left(\mathcal{L}_{x}\right)^{2}=1, \forall x \in X$, and the map $r$ has a concrete orbit of order 9 , say:

$$
\begin{aligned}
\mathcal{O}(98): \quad 98 & \rightarrow_{r} 79 \rightarrow_{r} 67 \rightarrow_{r} 56 \rightarrow_{r} 45 \\
& \rightarrow_{r} 34 \rightarrow_{r} 23 \rightarrow_{r} 12 \rightarrow_{r} 81 \rightarrow_{r} 98
\end{aligned}
$$

Then the orbit (6.10) determines the permutations $\mathcal{L}_{x}, x \in X$ uniquely, so that $(X, r)$ is a non-degenerate 2 -cancelative braided set, with $\mathcal{L}_{x}^{2}=1, x \in X$. More precisely,

(1) $(X, r)$ is a braided set iff the left actions satisfy

$$
\begin{aligned}
& \mathcal{L}_{9}=(16)(25)(34)(78) \quad \mathcal{L}_{8}=(19)(27)(36)(45) \\
& \mathcal{L}_{7}=(14)(23)(58)(69) \quad \mathcal{L}_{6}=(12)(38)(49)(57) \\
& \mathcal{L}_{5}=\left(\begin{array}{ll}
1 & 8)(29)(37)(46) \quad \mathcal{L}_{4}=(17)(26)(35)(89)
\end{array}\right. \\
& \mathcal{L}_{3}=(15)(24)(68)(97) \quad \mathcal{L}_{2}=(13)(48)(67)(59) \\
& \mathcal{L}_{1}=(28)(39)(56)(47)
\end{aligned}
$$

(2) In this case the non-trivial $r$ - orbits of $X^{2}$ are:

$\mathcal{O}(12), \mathcal{O}(18), \mathcal{O}(19) \mathcal{O}(13), \mathcal{O}(15), \mathcal{O}(16) \quad$ these are 6 orbits of order 9

$\mathcal{O}(29), \mathcal{O}(92), \mathcal{O}(14), \mathcal{O}(41), \mathcal{O}(36), \mathcal{O}(63) \quad$ these are 6 orbits of order 3

Moreover, $(X, r)$ decomposes as a union of three $r$-invariant subsets

$X=X_{1} \bigcup X_{2} \bigcup X_{3}, \quad X_{1}=\{1,4,7\}, X_{2}=\{3,6,8\}, X_{3}=\{2,5,9\}$,

and $r$ induces maps

$$
X_{1} \times X_{2} \longrightarrow X_{3} \times X_{1} \longrightarrow X_{2} \times X_{3} \longrightarrow X_{1} \times X_{2} .
$$

Each $\left(X_{i}, r_{i}\right), 1 \leq i \leq 3$ is an $\mathbf{S D}$ solution whose quandle is isomorphic to the dihedral quandle of order 3.

Next we give an example of an indecomposable square-free solution $(X, r)$ of order $|X|=4$ which fails to satisfy the minimality condition $\mathbf{M}$.

Example 6.21. Suppose $(X, r)$ is a square-free quadratic SD set of order $|X|=4$, so $r(x, y)=\left({ }^{x} y, x\right)$. We again simplify notation setting $X=\{1,2,3,4\}$. Suppose $\mathcal{L}_{4}$ is not involutive, and $\langle r\rangle$ has an orbit of length 3 say:

$$
\mathcal{O}(24): \quad 43 \longrightarrow_{r} 24 \longrightarrow_{r} 32 \longrightarrow_{r} 43 .
$$

The orbit (6.13) determines the maps $\mathcal{L}_{i}, i \in X$ (and $r$ ) uniquely, so that $(X, r)$ is a 2 -cancelative solution. More precisely,

(1) $(X, r)$ is a braided set iff the left actions are:

$$
\begin{array}{ll}
\mathcal{L}_{1}=\left(\begin{array}{lll}
3 & 4 & 2
\end{array}\right), & \mathcal{L}_{2}=\left(\begin{array}{lll}
4 & 3 & 1
\end{array}\right), \\
\mathcal{L}_{3}=\left(\begin{array}{lll}
2 & 4 & 1
\end{array}\right), & \mathcal{L}_{4}=\left(\begin{array}{lll}
3 & 2 & 1
\end{array}\right) .
\end{array}
$$

(2) In this case $X^{2}$ has $4 r$-orbits of length 3 (it is easy to write them explicitly), and 4 one-element orbits for the elements of $\operatorname{diag}\left(X^{2}\right)$.

(3) We consider the degree-lexicographic ordering $\leq$ on $\langle X\rangle$, induced by the ordering $1<2<3<4$ on $X$. The set of defining relations $\Re(r)$ reduces (and is equivalent) to the following set of 8 quadratic relations:

$$
\mathbf{R}=\left\{\begin{array}{llll}
43=24 & 32=24 & 42=14 & 21=14 \\
41=13 & 34=13 & 31=12 & 23=12 .
\end{array}\right.
$$


(4) Let $A=A(\mathbf{k}, X, r)=\mathbf{k}\langle X ; \mathbf{R}\rangle \cong \mathbf{k}\langle X\rangle /(I)$ be the associated quadratic algebra graded by length. The ideal $I$ is generated by the set

$$
\mathbf{R}_{0}=\{u-v \mid u=v \in \mathbf{R}\} \subset \mathbf{k}\langle X\rangle .
$$

It is not difficult to show that the reduced Gröbner basis $\mathbf{G B}\left(\mathbf{R}_{0}\right)$ contains 4 additional relations:

$\mathbf{G B}\left(\mathbf{R}_{0}\right)=\mathbf{R}_{0} \bigcup\{244-133,224-122,1444-1222,1333-1222\}$.

It follows that $A$ is standard finitely presented.

(5) The set $\mathcal{N}$ of normal $(\bmod I)$ monomials, which projects to a $\mathbf{k}$-basis of $A$ satisfies:

$$
\begin{gathered}
\mathcal{N} \supset \quad X \bigcup\{12,13,14,24\} \bigcup\{112,113,114,122,124,133,144\} \\
\bigcup\left\{1^{k} 2^{m}, k \geq 1, m \geq 3\right\} \bigcup\left\{x^{k} \mid x \in X, k \geq 2\right\} .
\end{gathered}
$$

(6) $\operatorname{dim}_{\mathbf{k}} A_{2}=8>2|X|-1$.

(7) $G K \operatorname{dim}_{A}=2$.

(8) $(X, r)$ is indecomposable, and injective, but $(X, r)$ does not satisfy the minimality condition $\mathbf{M}$.

(9) The monoid $S$ satisfies the relations (6.15) and also the following relations derived from the Gröbner basis

$$
244=133,224=122,1444=1222,1333=1222 .
$$

In particular $S$ is 3-cancellative, but $S$ is not cancellative .

(10) The group $G(X, r)$ satisfies the relations (6.15) which (only in the group case) give rise to the following new relations in $G: 444=333=222=111$.

\section{A CLASS OF SPECIAL EXTENSIONS}

Remark 7.1. Let $(X, r)$ be a quadratic set. A permutation $\tau \in \operatorname{Sym}(X)$ is called an automorphism of $(X, r)$ (or shortly an $r$-automorphism) if $(\tau \times \tau) \circ r=r \circ(\tau \times \tau)$. The group of $r$-automorphisms of $(X, r)$ is denoted by $\operatorname{Aut}(X, r)$.

In the hypothesis of the following theorem $\left(X, r_{X}\right),\left(Y, r_{Y}\right)$ are most general disjoint braided sets. No restrictions like nondegeneracy or 2-cancellativeness are imposed.

Theorem 7.2. Let $\left(X, r_{X}\right)$, and $\left(Y, r_{Y}\right)$ be disjoint braided sets, and let $Z=$ $X \bigcup Y$. Suppose $\sigma \in \operatorname{Sym}(X), \sigma \neq 1, \tau \in \operatorname{Sym}(Y), \tau \neq 1$. Define a bijective map $r: Z \times Z \longrightarrow Z \times Z$, as follows

$r(y, x):=(\sigma(x), \tau(y)) ; \quad r(x, y):=(\tau(y), \sigma(x)), \forall x \in X, y \in Y$.

$r\left(x_{1}, x_{2}\right):=r_{X}\left(x_{1}, x_{2}\right), \forall x_{1}, x_{2} \in X, \quad r\left(y_{1}, y_{2}\right):=r_{Y}\left(y_{1}, y_{2}\right), \forall y_{1}, y_{2} \in Y$,

Then $(Z, r)$ is a quadratic set which satisfies the following conditions.

(1) $(Z, r)$ is non-degenerate iff both $\left(X, r_{X}\right)$, and $\left(Y, r_{Y}\right)$ are non-degenerate.

(2) $(Z, r)$ is 2-cancellative iff (i) both $\left(X, r_{X}\right)$, and $\left(Y, r_{Y}\right)$ are 2-cancellative; and (ii) the maps $\sigma$ and $\tau$ (considered as permutations) are products of disjoint cycles of the same length $q$. Clearly, in this case $|\sigma|=|\tau|=q$.

(3) Suppose the conditions (2) are satisfied. For each pair $x \in X, y \in Y$ consider the r-orbit $\mathcal{O}(x y)=\left\{r^{k}(x y) \mid k \geq 0\right\}$ in $Z^{2}$.

(a) If $q$ is even, $q=2 m$, then $|\mathcal{O}(x y)|=q$. In this case the order $|r|$ of the map $r$ is the least common multiple of the three orders, $\boldsymbol{L C M}\left(\left|r_{X}\right|,\left|r_{Y}\right|, q\right)$. 
(b) If $q$ is odd, $q=2 m+1$ then $|\mathcal{O}(x y)|=2 q$. In this case the order $|r|$ of $r$ is the least common multiple $\boldsymbol{L} \boldsymbol{C M}\left(\left|r_{X}\right|,\left|r_{Y}\right|, 2 q\right)$.

(4) The quadratic set $(Z, r)$ is a regular extension of $\left(X, r_{X}\right)$, and $\left(Y, r_{Y}\right)$, in the sense of [19] if and only if $\sigma^{2}=\tau^{2}=1$. Moreover, $(Z, r)$ is involutive iff (i) $\sigma^{2}=\tau^{2}=1$, and (ii) $\left(X, r_{X}\right)$ and $\left(Y, r_{Y}\right)$ are involutive.

(5) $(Z, r)$ obeys the $Y B E$ if and only if the following conditions hold:

(a) $\sigma \in \operatorname{Aut}\left(X, r_{X}\right)$ and $\tau \in \operatorname{Aut}\left(Y, r_{Y}\right)$;

(b) The left and the right actions satisfy the following conditions.

$$
\begin{array}{lll}
\mathcal{L}_{\sigma^{2}(x)}=\mathcal{L}_{x}, & \mathcal{R}_{\sigma^{2}(x)}=\mathcal{R}_{x} & \text { hold in }\left(X, r_{X}\right), \forall x \in X . \\
\mathcal{L}_{\tau^{2}(y)}=\mathcal{L}_{y} & \mathcal{R}_{\tau^{2}(y)}=\mathcal{R}_{y} & \text { hold in }\left(Y, r_{Y}\right), \forall y \in Y .
\end{array}
$$

In this case $(Z, r)=\left(X, r_{X}\right) \natural^{*}\left(Y, r_{Y}\right)$ is a generalized strong twisted union of $X$ and $Y$ see Definition 8.7

Proof. Parts (1), (2), (3) and (4) are easy, and we leave their proof to the reader. We shall prove part (5). Assume $(Z, r)$ obeys YBE. We shall prove condition (a) and (b). Consider the diagram (7.2), where $\alpha \in Y, y, z \in X$. This diagram contains elements of the orbit of the monomial $\alpha y z \in Z^{3}$, under the action of the group $\mathcal{D}_{3}(r)$. Each two monomials in this orbit are equal as elements of $S$

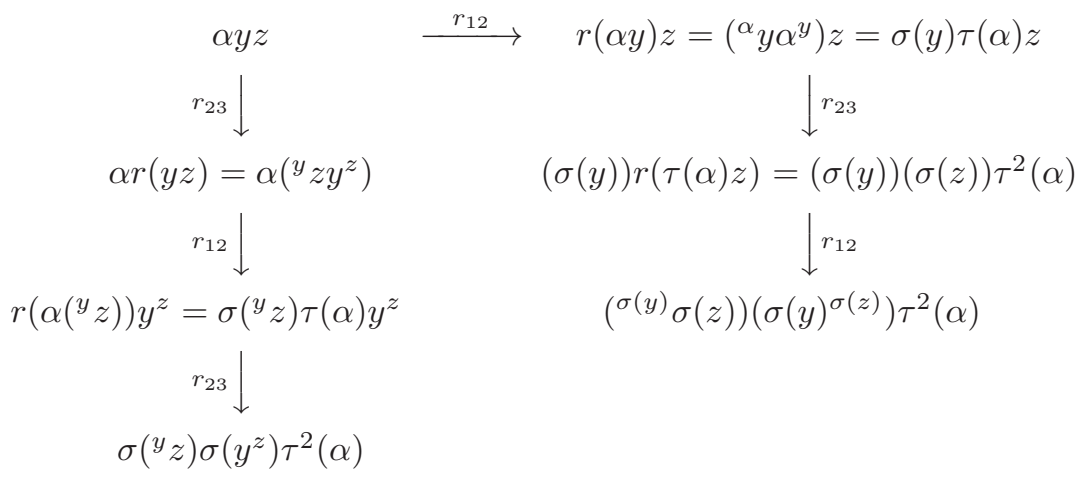

Therefore,

$$
\begin{aligned}
r_{12} r_{23} r_{12}(\alpha y z) & =r_{23} r_{12} r_{23}(\alpha y z), \quad \forall \alpha \in Y, y, z \in X \\
& \Longleftrightarrow(\sigma \times \sigma) \circ r_{X}(y z)=r_{X} \circ(\sigma \times \sigma)(y z), \forall y, z \in X \\
& \Longleftrightarrow \sigma \in \operatorname{Aut}\left(X, r_{X}\right) .
\end{aligned}
$$

Similarly, a diagram starting with an arbitrary monomial of the shape $x \alpha \beta$, where $x \in X, \alpha, \beta \in Y$ shows that

$$
\begin{aligned}
r_{12} r_{23} r_{12}(x \alpha \beta) & =r_{23} r_{12} r_{23}(x \alpha \beta) \quad \forall x \in X, \alpha, \beta \in Y \\
& \Longleftrightarrow(\tau \times \tau) \circ r_{Y}(\alpha \beta)=r_{Y} \circ(\tau \times \tau)(\alpha \beta), \forall \alpha, \beta \in Y \\
& \Longleftrightarrow \tau \in \operatorname{Aut}\left(Y, r_{Y}\right) .
\end{aligned}
$$

We have proven (a). Next we shall prove (7.1). Consider the following diagram. 


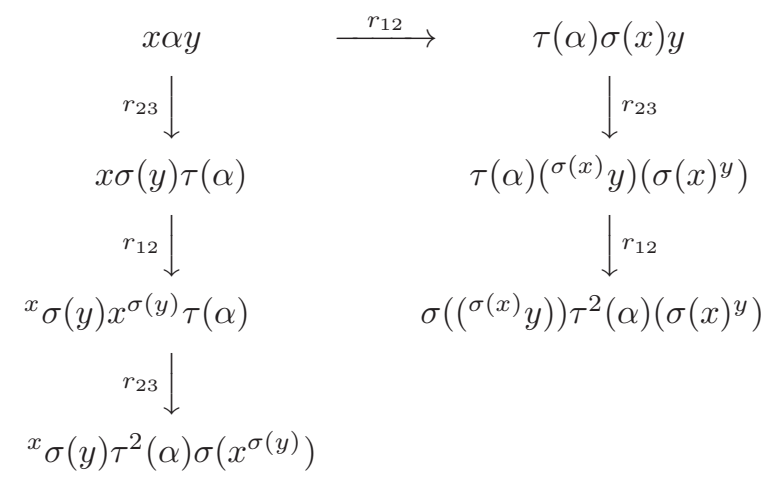

Hence

$$
\begin{aligned}
& r_{12} r_{23} r_{12}(x \alpha y)=r_{23} r_{12} r_{23}(x \alpha y), \quad \forall x, y \in X, \alpha \in Y \\
& \Longleftrightarrow \sigma\left(\left({ }^{\sigma(x)} y\right)\right)={ }^{x}(\sigma(y)) \text { and } \sigma\left(x^{(\sigma(y))}\right)=\sigma(x)^{y}, \forall x, y \in X .
\end{aligned}
$$

But $\sigma \in \operatorname{Aut}\left(X, r_{X}\right)$ so it follows from (7.6) that

$$
{ }^{x}(\sigma(y))=\sigma\left(\left({ }^{\sigma(x)} y\right)\right)={ }^{\sigma(\sigma(x))} \sigma(y)={ }^{\left(\sigma^{2}(x)\right)}(\sigma(y)), \forall x, y \in X .
$$

The map $\sigma: X \longrightarrow X$ is bijective, hence

$$
{ }^{\left(\sigma^{2}(x)\right)} z={ }^{x} z, \forall x, z \in X,
$$

which is equivalent to

$$
\mathcal{L}_{\sigma^{2}(x)}=\mathcal{L}_{x} \text { holds in }\left(X, r_{X}\right), \forall x \in X .
$$

Similarly, the equalities

$$
(\sigma(x))^{\left(\sigma^{2}(y)\right)}=\sigma\left(x^{\sigma(y)}\right)=(\sigma(x))^{y}, \forall x, y \in X
$$

are equivalent to

$$
\mathbf{R}_{\sigma^{2}(y)}=\mathbf{R}_{y} \text { holds in }\left(X, r_{X}\right), \forall y \in X .
$$

This proves the first two equalities in (7.1). Analogous argument proves the remaining equalities in (7.1). We have shown that if $(Z, r)$ obeys YBE then conditions (a) and (b) hold. Conversely, assume that conditions (a) and (b) are satisfied. The above discussion implies easily that $(Z, r)$ is a solution of YBE. This proves part (5).

It is clear that our construction gives a particular case of a generalized strong twisted union $(Z, r)=\left(X, r_{X}\right) \natural^{*}\left(Y, r_{Y}\right)$, see Definition 8.7

One may apply the results of Theorem 8.12 and get more information on the braided monoid $S(Z, r)$ and the braided group $G(Z, r)$.

To construct concrete extensions via automorphisms, and also for some kind of classification of this type of extensions it may be practical to use results from 8 .

Clearly, if $(X, r)$ is a trivial solution, then $\operatorname{Aut}(X, r)=\operatorname{Sym}(X)$ and for every $\sigma \in \operatorname{Sym}(X)$ there is an equality $\mathcal{L}_{\sigma^{2}(x)}=\mathcal{L}_{x}=\operatorname{id}_{X}$. Hence we have at disposal an easy method to construct non-degenerate 2 -cancellative square-free braided sets $(Z, r), Z=X \bigcup Y$, where the order of the map $r$ may vary as $2 \leq|r| \leq|Z|$. 
Corollary 7.3. Let $\left(X, r_{X}\right),\left(Y, r_{Y}\right)$ be disjoint trivial symmetric sets, $|X|=$ $m,|Y|=n$, say $m \leq n$. Let $Z=X \bigcup Y$. Suppose $\sigma \in \operatorname{Sym}(X), \tau \in \operatorname{Sym}(Y)$. Define $r: Z \times Z \longrightarrow Z \times Z$, as follows

$r\left(x_{1}, x_{2}\right):=r_{X}\left(x_{1}, x_{2}\right)=\left(x_{2}, x_{1}\right), \forall x_{1}, x_{2} \in X, \quad r\left(y_{1}, y_{2}\right):=r_{Y}\left(y_{1}, y_{2}\right)=\left(y_{2}, y_{1}\right), \forall y_{1}, y_{2} \in Y$, $r(x, y):=(\tau(y), \sigma(x)), \quad r(y, x):=(\sigma(x), \tau(y)) ; \forall x \in X, y \in Y$.

(1) $(Z, r)$ is a non-degenerate square-free braided set.

(2) Moreover, $(Z, r)$ is 2-cancellative iff the permutations $\sigma$ and $\tau$ are products of disjoint cycles of the same length $q \leq m$, in particular, $|\sigma|=|\tau|=q$; In this case either (a) $q$ is even, and $|r|=q$; or (b) $q$ is odd and $|r|=2 q$.

Example 7.4. Let $X=\left\{x_{1}, x_{2}, x_{3}\right\}, Y=\left\{y_{1}, y_{2}, y_{3}\right\}$, be disjoint sets and let $\left(X, r_{X}\right),\left(Y, r_{Y}\right)$ be trivial solutions.

Set $\sigma=\left(\begin{array}{lll}x_{1} & x_{2} & x_{3}\end{array}\right) \in \operatorname{Sym}(X), \tau=\left(\begin{array}{lll}y_{1} & y_{2} & y_{3}\end{array}\right) \in \operatorname{Sym}(Y)$. Define $r: Z \times Z \longrightarrow$ $Z \times Z$, as follows

$$
\begin{array}{ll}
r\left(x_{i}, x_{j}\right)=\left(x_{j}, x_{i}\right), & r\left(y_{i}, y_{j}\right)=\left(y_{j}, y_{i}\right), 1 \leq i, j \leq 3, \\
r(y, x)=(\sigma(x), \tau(y)), & r(x, y)=(\tau(y), \sigma(x)), \forall x \in X, y \in Y .
\end{array}
$$

Then $(Z, r)$ is a non-degenerate square-free braided set of order $|Z|=6, Z=X \natural^{*} Y$. $(Z, r)$ is 2-cancellative. The order of $r$ is $|r|=6=|Z|$. More detailed computation shows that the associated graded algebra $A$ does not have a finite Gröbner basis with respect to any ordering of $Z$.

\section{The BRAided Monoid $S(X, r)$ And EXTEnsions of SOlutions}

8.1. The braided monoid $S(X, r)$ of a braided set $(X, r)$. In [19] we have defined a braided monoid analogously to the term 'braided group' in the sense of [36], 25. We recall some notions and results from [19.

To each braided set $(X, r)$ we associate a matched pair $(S, S)(S=S(X, r))$ with left and right actions uniquely determined by $r$, which defines a unique 'braided monoid' $\left(S, r_{S}\right)$ associated to $(X, r)$. This is not a surprise given the analogous results for the group $G(X, r)$, see [25], but our approach is necessarily different. In fact we first construct the matched pair or monoids ([19], Theorem 3.6) which is a self-contained result and then consider $r_{S}$. We prove, see [19] Theorem 3.14, that $r_{S}$ is bijective and obeys the YBE (as would be true in the group case), moreover, we show that $\left(S, r_{S}\right)$ is braided monoid.

The reader should be aware that due to the possible lack of cancellation in $S$ the proofs of our results for monoids are difficult and necessarily involve different computations and combinatorial arguments. Surely, the results can not be extracted from the already known results from the group case. Nevertheless, the monoid case is the one naturally arising in this context. Both the monoid $S(X, r)$ and the quadratic algebra $A=A(\mathbf{k}, X, r)$ over a field $\mathbf{k}$ are of particular interest. The theory of general braided monoids $\left(S, r_{S}\right)$ gives interesting classes of braided objects, however it seems that the approach to these is different and more difficult from the approach to braided groups (equivalently skew braces).

We recall some basic definitions.

Definition 8.1. 19] The pair $(S, T)$ is a matched pair of monoids if $T$ acts from the left on $S$ by ${ }^{()} \bullet$ and $S$ acts on $T$ from the right by $\bullet$ ( ) and these two actions 
obey

$$
\begin{array}{cccc}
\text { ML0 : } & { }^{a} 1=1, \quad{ }^{1} u=u ; & \text { MR0 : } & 1^{u}=1, \quad a^{1}=a \\
\text { ML1 : } & (a b) u={ }^{a}\left({ }^{b} u\right), & \text { MR1 : } & a^{(u v)}=\left(a^{u}\right)^{v} \\
\text { ML2 : } & { }^{a}(u . v)=\left({ }^{a} u\right)\left({ }^{a} v\right), & \text { MR2 : } & (a . b)^{u}=\left(a^{b} u\right)\left(b^{u}\right),
\end{array}
$$

for all $a, b \in T, u, v \in S$.

Definition 8.2. 19] An M3-monoid is a monoid $S$ forming part of a matched pair $(S, S)$ for which the actions are such that

$$
\text { M3: }{ }^{u} v u^{v}=u v
$$

holds in $S$ for all $u, v \in S$. We define the associated map $r_{S}: S \times S \rightarrow S \times S$ by $r_{S}(u, v)=\left({ }^{u} v, u^{v}\right)$. A braided monoid is an M3-monoid $S$, where $r_{S}$ is bijective and obeys the YBE.

Fact 8.3. (19, Theor. 3.6, Theor. 3.14.) Let $(X, r)$ be a braided set and $S=$ $S(X, r)$ the associated monoid. Then

(1) The left and the right actions ( ) $\bullet: X \times X \longrightarrow X$, and $\bullet$ ( ) $: X \times X \longrightarrow X$ defined via $r$ can be extended in a unique way to a left and a right action

$$
\text { ( ) }: S \times S \longrightarrow S \text {, and •( ) }: S \times S \longrightarrow S \text {. }
$$

which make $S$ a strong graded M3-monoid. The associated bijective map $r_{S}$ restricts to $r$.

(2) Moreover, the following conditions hold.

(a) $\left(S, r_{S}\right)$ is a graded braided monoid.

(b) $\left(S, r_{S}\right)$ is a non-degenerate solution of $Y B E$ iff $(X, r)$ is non-degenerate.

(c) $\left(S, r_{S}\right)$ is involutive iff $(X, r)$ is involutive.

Note that by construction, the actions agree with the grading of $S$, i.e. $\left|{ }^{a} u\right|=$ $|u|=\left|u^{a}\right|$, for all $a, u \in S$.

Suppose $(X, r)$ is a noninvolutive solution. The set $X$ is always embedded in the braided monoid $\left(S, r_{S}\right)$. Moreover, in contrast with the group $G(X, r)$ the monoid $S$ preserves more detailed information about the solution. In particular, there is an equality $u=v$ in $S$, if and only if $|u|=|v|=m$, and $u$ and $v$ are in the same $\mathcal{D}_{m}(r)$-orbit in $X^{m}$, but in general, this is not true in $G(X, r)$, where a great portion of information about $(X, r)$ is lost.

\subsection{General extensions of braided sets.}

Definition 8.4. Let $\left(X, r_{X}\right)$ and $\left(Y, r_{Y}\right)$ be disjoint quadratic sets. Let $(Z, r)$ be a set with a bijection $r: Z \times Z \longrightarrow Z \times Z$. We say that $(Z, r)$ is a (general) extension of $\left(X, r_{X}\right),\left(Y, r_{Y}\right)$, if $Z=X \bigcup Y$ as sets, and $r$ extends the maps $r_{X}$ and $r_{Y}$, i.e. $r_{\mid X^{2}}=r_{X}$, and $r_{\mid Y^{2}}=r_{Y}$. Clearly in this case $X, Y$ are $r$-invariant subsets of $Z$. $(Z, r)$ is a $Y B$-extension of $\left(X, r_{X}\right)$, and $\left(Y, r_{Y}\right)$ if $r$ obeys YBE.

Remark 8.5. In the assumption of the above definition, suppose $(Z, r)$ is a nondegenerate extension of $\left(X, r_{X}\right),\left(Y, r_{Y}\right)$. Then the equalities $r(x, y)=\left({ }^{x} y, x^{y}\right)$, $r(y, x)=\left({ }^{y} x, y^{x}\right)$, and the nondegeneracy of $r, r_{X}, r_{Y}$ imply that

$$
{ }^{y} x, x^{y} \in X \text {, and }{ }^{x} y, y^{x} \in Y \text {, for all } x \in X, y \in Y \text {. }
$$

Therefore, $r$ induces bijective maps

$$
\rho: Y \times X \longrightarrow X \times Y \text {, and } \sigma: X \times Y \longrightarrow Y \times X,
$$


and left and right "actions"

$$
\begin{gathered}
{ }^{Y}: Y \times X \longrightarrow X, \bullet^{X}: Y \times X \longrightarrow Y \text {, projected from } \rho \\
{ }^{X} \bullet: X \times Y \longrightarrow Y, \quad \bullet^{Y}: X \times Y \longrightarrow X, \text { projected from } \sigma .
\end{gathered}
$$

Clearly, the 4-tuple of maps $\left(r_{X}, r_{Y}, \rho, \sigma\right)$ uniquely determine the extension $r$. The map $r$ is also uniquely determined by $r_{X}, r_{Y}$, and the maps (8.2), (8.3).

We call the actions 8.2), (8.3) projected from $r_{\mid Y \times X}$ and $r_{\mid X \times Y}$ the associated ground actions.

Lemma 8.6. Suppose $(Z, r)$ is a non-degenerate braided set which splits as a disjoint union $Z=X \bigcup Y$ of two $r$-invariant subsets $X$ and $Y$, denote by $\left(X, r_{1}\right)$ and $\left(Y, r_{2}\right)$ the induced sub-solutions. The following conditions hold.

(1) The assignment $\alpha \longrightarrow{ }^{\alpha} \bullet=\mathcal{L}_{\alpha \mid X}$ extends to a left action of $G_{Y}$ (and the associated monoid $S_{Y}$ ) on $X$, the assignment $\alpha \longrightarrow \bullet^{\alpha}=\boldsymbol{R}_{\alpha \mid X}$ extends to a right action of $G_{Y}$ (and the associated monoid $S_{Y}$ ) on $X$.

(2) The assignment $x \longrightarrow{ }^{x} \bullet=\mathcal{L}_{x \mid Y}$ extends to a left action of $G_{X}$ (and the associated monoid $\left.S_{X}\right)$ on $Y$, the assignment $x \longrightarrow \bullet^{x}=\boldsymbol{R}_{x \mid Y}$ extends to a right action of $G_{X}$ (and the associated monoid $S_{X}$ ) on $Y$.

Recall that in [19] a (general) extension $(Z, r)$ of $\left(X, r_{X}\right),\left(Y, r_{Y}\right)$ is called $a$ regular extension of $\left(X, r_{X}\right)$, and $\left(Y, r_{Y}\right)$ if $r$ is bijective, and the restrictions $r_{\mid Y \times X}$ and $r_{\mid X \times Y}$ satisfy

$$
(r \circ r)_{\mid Y \times X}=\operatorname{id}_{\mid Y \times X}, \quad(r \circ r)_{\mid X \times Y}=\operatorname{id}_{\mid X \times Y},
$$

but $r$ is not necessarily involutive on $X \times X$, neither on $Y \times Y$. Regular extensions of arbitrary braided sets were introduced and studied in [19], where the theory of matched pairs of monoids was applied to characterize regular extensions and their monoids. Note that a regular extension $(Z, r)$ of two involutive solutions is also involutive.

In this paper we have a particular interest in noninvolutive non-degenerate braided sets $(Z, r)$, and it is natural to search for methods proposing constructions of "new" solutions using already known braided sets. We have shown in Section 7. see Theorem 7.2 that one can construct new noninvolutive solutions $(Z, r)$ with a prescribed order $|Z|$, using general (non-regular) extensions of well-known involutive solutions. So it is natural to study general extensions $(Z, r)$, which are not necessarily regular in the sense of [19). In notation and assumptions as above, let $(Z, r)$ be a non-degenerate braided set which is an extension of the disjoint braided sets $\left(X, r_{X}\right),\left(Y, r_{Y}\right)$. Denote $S=S\left(X, r_{X}\right), T=S\left(Y, r_{Y}\right), U=S(Z, r)$. It follows from Fact 8.3 that $U=S\left(Z, r_{Z}\right)$ has the structure of a graded braided monoid $\left(U, r_{U}\right)$ with a braiding operator $r_{U}$ extending $r$, moreover $\left(U, r_{U}\right)$ is an extension of the disjoint braided monoids $\left(S, r_{S}\right)$ and $\left(T, r_{T}\right)$, and one can apply the theory of matched pairs of monoids to give more detailed description of the behaviour of the matched pairs $(\mathrm{S}, \mathrm{T}),(\mathrm{T}, \mathrm{S}),(U, U)$, etc, in the spirit of the results in 19]).

We shall propose an explicit construction- generalized strong twisted unions of braided sets. 
8.3. Generalized strong twisted unions of non-degenerate braided sets. Theorem ?? gives a method to construct new type of extensions of braided sets. The properties of these extensions motivate our new Definition 8.7 of generalized strong twisted unions of solutions which is a generalization of our previous definition, see Definition 5.1. 19]. According the "old" definition the notion of strong twisted union is restricted only to regular extensions. Note that a strong twisted union $(Z, r)$ of solutions $\left(X, r_{X}\right)$ and $\left(Y, r_{Y}\right)$ does not necessarily obey YBE, but if $(Z, r)=X \sharp Y$ is (a regular) extension of symmetric sets and obeys the YBE, then $(Z, r)$ is also a symmetric set $\left(r^{2}=1\right)$.

In our new settings if $\left(X, r_{X}\right)$ and $\left(Y, r_{Y}\right)$ are symmetric (or braided) sets with $|X|>2,|Y|>2$ we construct extensions $(Z, r)$ which are braided sets (satisfy $\mathrm{YBE}$ ), but the solution $r$ may have order $>2$, see for example section 7 and the results therein.

Definition 8.7. Suppose $\left(X, r_{X}\right)$ and $\left(Y, r_{Y}\right)$ are disjoint quadratic sets. We call an extension $(Z, r)$ a generalized strong twisted union of $\left(X, r_{X}\right)$ and $\left(Y, r_{Y}\right)$, and write $Z=X \natural^{*} Y$ if the ground actions satisfy

$$
\begin{aligned}
& \text { stu1: } \quad{ }^{y} x={ }^{\alpha} x ; \quad \text { stu2: } \quad x^{y} \alpha=x^{\alpha} ; \\
& \text { stu3 : } \quad x^{\beta} \alpha={ }^{x} \alpha ; \quad \text { stu } 4: \quad \alpha^{\beta} x=\alpha^{x}
\end{aligned}
$$

for all $x, y \in X, \alpha, \beta \in Y$.

Similarly, to [18, Definition 3.5, one defines generalized strong twisted union of more than 2 quadratic sets as follows. Let $(X, r)$ be a nondegenerate quadratic set (of arbitrary cardinality), let $X_{i}, i \in I$, be a set of pairwise disjoint r-invariant subsets, ( $I$ is a set of indices). We say that $(X, r)$ is a generalized strong twisted union of $X_{i}, i \in I$ and write $X=\natural_{i \in I}^{*} X_{i}$ if it is a (disjoint) union $X=\bigcup_{i \in I} X_{i}$, and for each pair $i, j \in I, i \neq j$ the $\mathrm{r}$ - invariant subset $X_{i j}=X_{i} \bigcup X_{j}$ is a generalized strong twisted union, $X_{i j}=X_{i} \natural^{*} X_{j}$. In the particular case, when $I$ is a finite set $\{1 \leq i \leq m\}$, we write $X=X_{1} \natural^{*} X_{2} \natural^{*} \cdots \natural^{*} X_{m}$.

Remark 8.8. Suppose $(X, r)$ is an SD non-degenerate braided set i.e. $r(x, y)=$ $\left({ }^{x} y, x\right), \forall x, y \in X$ (so $(X, \triangleright)$ is a quandle). If $(X, r)$ decomposes as a union of disjoint $r$-invariant subsets $X=Y \cup Z$, then $X=Y \natural^{*} Z$.

Lemma 8.9. Let $(Z, r)$ be a non-degenerate quadratic set, which splits as a disjoint union $Z=X \bigcup Y$ of its $r$-invarint subsets $\left(X, r_{X}\right),\left(Y, r_{Y}\right)$, (so $Z$ is an extension of $X$ and $Y)$. Suppose $x, y \in X$, alpha $\in Y$. Each two of the following conditions imply the third.

$$
\begin{array}{lll}
\text { (1) } \quad \text { l1 }(\alpha, \mathbf{y}, \mathbf{x}): & { }^{\alpha}\left({ }^{y} x\right)={ }^{\alpha} y\left(\alpha^{y} x\right) \\
\text { (2) } \quad \operatorname{laut}(\alpha, \mathbf{y}, \mathbf{x}): & { }^{\alpha}\left({ }^{y} x\right)={ }^{\alpha} y\left({ }^{\alpha} x\right) \\
\text { (3) } \quad \operatorname{stu1}(\alpha, \mathbf{y}, \mathbf{x}): & { }^{y} x={ }^{\alpha} x .
\end{array}
$$

Let $(Z, r)$ be a non-degenerate braided set which split as a disjoint union $Z=$ $X \bigcup Y$ of two $r$-invariant subsets $X$ and $Y, G_{Z}=G(Z, r)$. Denote by $\left(X, r_{X}\right)$ and $\left(Y, r_{Y}\right)$ the induced subsolutions. Due to the nondegeneracy of $r$, each of the sets $X$ and $Y$ is invariant under the left action of $G_{Z}$ on $Z$, similarly, $X$ and $Y$ are invariant under the right action of $G_{Z}$ on $Z$. (We call such sets $G$-invriant). Let $\alpha \in Y$, and 
let $\mathcal{L}_{\alpha}$ be the corresponding left action on $Z$. Denote by $\mathcal{L}_{\alpha \mid X}, \alpha \in Y$ the restriction of $\mathcal{L}_{\alpha}$ on $X$. The restrictions $\mathcal{R}_{\alpha \mid X}, \mathcal{L}_{x \mid Y}, \mathcal{R}_{x \mid Y}$ are defined analogously.

Proposition 8.10. Suppose $(Z, r)$ is a non-degenerate braided set which splits as a disjoint union $Z=X \bigcup Y$ of two $r$-invariant subsets $X$ and $Y$, denote by $\left(X, r_{1}\right)$ and $\left(Y, r_{2}\right)$ the induced subsolutions. The following conditions hold.

(1) $\mathcal{L}_{\alpha \mid X} \in \operatorname{Aut}\left(X, r_{1}\right)$ if and only if

$$
{ }^{\alpha}\left({ }^{y} x\right)={ }^{\alpha} y\left({ }^{\alpha} x\right), \text { and }{ }^{\alpha}\left(x^{y}\right)=\left({ }^{\alpha} x\right)^{\left({ }^{\alpha} y\right)}, \forall x, y \in X .
$$

(2) $\mathcal{R}_{\alpha \mid X} \in \operatorname{Aut}\left(X, r_{1}\right)$ if and only if

$$
\left({ }^{y} x\right)^{\alpha}={ }^{\left(y^{\alpha}\right)}\left(x^{\alpha}\right), \text { and }\left(x^{y}\right)^{\alpha}=\left(x^{\alpha}\right)^{\left(y^{\alpha}\right)}, \forall x, y \in X .
$$

(3) The following implications hold

$$
\begin{array}{lll}
\text { stu1: } & \alpha^{y} x={ }^{\alpha} x, \forall \alpha \in Y, x, y \in X \Longleftrightarrow & \mathcal{L}_{\alpha \mid X} \in \operatorname{Aut}\left(X, r_{1}\right), \forall \alpha \in Y \\
\text { stu2: } & x^{y} \alpha=x^{\alpha}, \forall \alpha \in Y, x, y \in X \Longleftrightarrow & \mathcal{R}_{\alpha \mid X} \in \operatorname{Aut}\left(X, r_{1}\right), \forall \alpha \in Y \\
\text { stu3: } & x^{\beta} \alpha={ }^{x} \alpha, \forall x \in X, \alpha, \beta \in Y \Longleftrightarrow & \mathcal{L}_{x \mid Y} \in \operatorname{Aut}\left(Y, r_{2}\right), \forall x \in X \\
\text { stu4: } & \alpha^{\beta}{ }^{\beta}=\alpha^{x}, \forall x \in X, \alpha, \beta \in Y \Longleftrightarrow & \mathcal{R}_{x \mid Y} \in \operatorname{Aut}\left(Y, r_{2}\right), \forall x \in X .
\end{array}
$$

Proof. (1). By definition $\mathcal{L}_{\alpha \mid X} \in \operatorname{Aut}\left(X, r_{X}\right)$ iff

$$
\left(\mathcal{L}_{\alpha \mid X} \times \mathcal{L}_{\alpha \mid X}\right) \circ r=r \circ\left(\mathcal{L}_{\alpha \mid X} \times \mathcal{L}_{\alpha \mid X}\right),
$$

so part (1) follows straightforwardly from the equalities in $X^{2}$ given below:

$$
\begin{aligned}
& \left(\mathcal{L}_{\alpha \mid X} \times \mathcal{L}_{\alpha \mid X} \circ r\right)(x, y)=\left({ }^{\alpha}\left({ }^{x} y\right),{ }^{\alpha}\left(x^{y}\right)\right) \\
& r \circ\left(\mathcal{L}_{\alpha \mid X} \times \mathcal{L}_{\alpha \mid X}\right)(x, y)=\left({ }^{x}\left({ }^{\alpha} y\right),\left({ }^{\alpha} x\right)^{\left({ }^{\alpha} y\right)}\right), \alpha \in Y, x, y \in X .
\end{aligned}
$$

Part (2) is analogous.

(3). We shall prove the first implication

$$
\text { stu1: } \quad \alpha^{y} x={ }^{\alpha} x, \forall \alpha \in Y, x, y \in X \Longleftrightarrow \mathcal{L}_{\alpha \mid X} \in \operatorname{Aut}\left(X, r_{1}\right), \forall \alpha \in Y .
$$

Recall first that the braided set $(Z, r)$ satisfies conditions 11 , lr3, see Remark 2.2

stu $1 \Longrightarrow \mathcal{L}_{\alpha \mid X} \in \operatorname{Aut}\left(X, r_{1}\right)$. Assume stu 1 holds in $Z$. This is condition (3) of Lemma 8.9. Note that $(Z, r)$ satisfies $\mathbf{1 1}$, and therefore condition (1) in Lemma 8.9 is also satisfied. Hence by Lemma 8.9 the remaining condition (2) is also in force. This gives

$$
{ }^{\alpha}\left({ }^{y} x\right)={ }^{\alpha} y\left({ }^{\alpha} x\right), \quad \forall \alpha \in Y, x, y \in X
$$

We shall prove

$$
{ }^{\alpha}\left(x^{y}\right)=\left({ }^{\alpha} x\right)^{\left({ }^{\alpha} y\right)}, \quad \forall \alpha \in Y, x, y \in X .
$$

We use $\mathbf{l r} 3$ and stu1 to deduce the following equalities

$$
\begin{aligned}
& \left.\left({ }^{\alpha} x\right)^{\left(\alpha^{\alpha^{x}} y\right)}={ }^{\left(\alpha^{x} y\right.}\right)\left(x^{y}\right) \quad \text { : by } \operatorname{lr} 3 \\
& { }^{\left(\alpha^{x y}\right)}\left(x^{y}\right)={ }^{\alpha}\left(x^{y}\right) \quad \text { : by stu1 } \\
& \left({ }^{\alpha} x\right)^{\left({ }^{\alpha^{x}} y\right)}=\left({ }^{\alpha} x\right)^{\left({ }^{\alpha} y\right)} \quad \text { : by stu1, }
\end{aligned}
$$

which imply (8.8). Hence $\mathcal{L}_{\alpha \mid X} \in \operatorname{Aut}\left(X, r_{1}\right), \forall \alpha \in Y$.

$\mathcal{L}_{\alpha \mid X} \in \operatorname{Aut}\left(X, r_{1}\right) \Longrightarrow$ stu 1. Suppose $\mathcal{L}_{\alpha \mid X} \in \operatorname{Aut}\left(X, r_{1}\right)$. By part (1) of our proposition this implies

$$
{ }^{\alpha}\left({ }^{y} x\right)={ }^{\alpha} y\left({ }^{\alpha} x\right), \forall x, y \in X,
$$


which is exactly condition (2) of Lemma 8.9. Condition (1) of Lemma 8.9 holds, this is $\mathbf{l 1}$, and therefore, the remaining condition (3) of Lemma 8.9 is also satisfied, but this is exactly stu $\mathbf{1}$. We have proven the equivalence (8.6). Analogous argument proves the remaining three equivalences in (8.5).

Lemma 8.6 and Proposition 8.10 imply straightforwardly the following.

Corollary 8.11. Suppose $(Z, r)$ is a non-degenerate 2-cancellative braided set which split as a disjoint union $Z=X \bigcup Y$ of its $r$-invariant subsets $X$ and $Y$. Let $\left(X, r_{1}\right)$ and $\left(Y, r_{2}\right)$ be the induced subsolutions. We assume that $\left(X, r_{1}\right)$ and $\left(Y, r_{2}\right)$ are injective. Then $(Z, r)=X \natural^{*} Y$ is a (generalized) strong twisted union if and only if the following four conditions hold.

(i) The assignment $x \longmapsto \mathcal{L}_{x \mid Y}$ extends to a group homomorphism

$$
\mathcal{L}_{X \mid Y}: G_{X} \longrightarrow \operatorname{Aut}\left(Y, r_{Y}\right),
$$

(ii) The assignment $x \longrightarrow \mathcal{R}_{x \mid Y}$ extends to a group homomorphism

$$
\mathcal{R}_{X \mid Y}: G_{X} \longrightarrow \operatorname{Aut}\left(Y, r_{Y}\right) \text {. }
$$

(iii) The assignment $\alpha \longrightarrow \mathcal{L}_{\alpha \mid X}$ extends to a group homomorphism

$$
\mathcal{L}_{Y \mid X}: G_{Y} \longrightarrow \operatorname{Aut}(X, r),
$$

(iv) The assignment $\alpha \longrightarrow \mathcal{R}_{\alpha \mid X}$ extends to a group homomorphism

$$
\mathcal{R}_{Y \mid X}: G_{Y} \longrightarrow \operatorname{Aut}(X, r) .
$$

Theorem 8.12. Suppose $(Z, r)$ is a non-degenerate 2-cancellative braided set which splits as a generalized strong twisted union $Z=X \natural^{*} Y$ of its $r$-invariant subsets $X$ and $Y$. Let $\left(X, r_{1}\right)$ and $\left(Y, r_{2}\right)$ be the induced subsolutions, $S=S\left(X, r_{1}\right)$, $T=S\left(Y, r_{2}\right), U=S(Z, r)$ in usual notation. Let $\left(S, r_{S}\right),\left(T, r_{T}\right),\left(U, r_{U}\right)$ be the corresponding braided monoids, see Fact 8.3. Let $\left(G_{Z}, r_{G_{Z}}\right),\left(G_{X}, r_{G_{X}}\right),\left(G_{Y}, r_{G_{Y}}\right)$ be the associated braided groups. Then the following conditions hold.

(1) The braided monoid $\left(U, r_{U}\right)$ has a canonical structure of a generalized strong twisted union

$$
\left(U, r_{U}\right)=\left(S, r_{S}\right) \mathfrak{\natural}^{*}\left(T, r_{T}\right),
$$

extending the ground actions of the strong twisted union $Z=X \natural^{*} Y$.

(2) Suppose $(Z, r)$ is injective (i.e. $Z$ is embedded in $\left.G_{Z}\right)$. Then $\left(X, r_{1}\right)$ and $\left(Y, r_{2}\right)$ are also injective and the braided group $\left(G_{Z}, r_{G_{Z}}\right)$ has a canonical structure of a strong twisted union

$$
\left(G_{Z}, r_{G_{Z}}\right)=\left(G_{X}, r_{G_{X}}\right) \natural^{*}\left(G_{Y}, r_{G_{Y}}\right) .
$$

Proof. (1). It follows from Fact 8.3 that $U=S\left(Z, r_{Z}\right)$ has the structure of a graded braided monoid $\left(U, r_{U}\right)$ with a braiding operator $r_{U}$ extending $r$, moreover $\left(U, r_{U}\right)$ is a (general) extension of the disjoint braided monoids $\left(S, r_{S}\right)$ and $\left(T, r_{T}\right)$. We have to show that the four stu conditions are satisfied, see (8.4). We shall use induction on lengths of words to prove

$$
\text { stu1: } \quad u^{b} a={ }^{u} a, \forall u \in T, a, b \in S .
$$

Step 1. First we prove (8.9) for all $a \in S, b=y \in X, u=\alpha \in Y$ by induction on the length $|a|$ of $a$. Condition stu 1 on $Z$ gives the base for the induction. Assume (8.9) is true for all $u \in Y, b \in X$ and all $a \in S$, with $|a| \leq n$. Suppose 
$a \in S,|a|=n+1, u=\alpha \in Y, b=y \in X$. Then $a=t c$ where $c \in S,|c|=n, t \in X$, and the following equalities hold in $U$.

$$
\begin{aligned}
\alpha^{y} a={ }^{\alpha^{y}}(t c) & =\left({ }^{\alpha^{y}} t\right)^{\left(\alpha^{y}\right)^{t} c} & & : \text { by ML2 } \\
& =\left({ }^{\alpha} t\right)^{\left(\alpha^{y}\right)} c & & : \text { by stu } 1 \text { and } \mathrm{IH} \\
& =\left({ }^{\alpha} t\right)\left({ }^{\alpha} c\right) & & : \text { by stu } 1 \text { and } \mathrm{IH} .
\end{aligned}
$$

where $\mathrm{IH}$ is the inductive assumption.

$$
\begin{aligned}
{ }^{\alpha} a={ }^{\alpha}(t c) & =\left({ }^{\alpha} t\right)^{\left(\alpha^{t}\right)} c & & \text { : by ML2 } \\
& =\left({ }^{\alpha} t\right)\left({ }^{\alpha} c\right) & & \text { : by stu } 1 \text { and } \mathrm{IH} .
\end{aligned}
$$

Equalities (8.10) and (8.11) imply ${ }^{\alpha^{y}} a={ }^{\alpha} a$, and therefore

$$
{ }^{\alpha^{y}} a={ }^{\alpha} a \quad \forall a \in S, \forall y \in X, \alpha \in Y .
$$

Step 2. We use induction on the length $|u|$ of $u \in T$ to prove

$$
u^{u^{y}} a={ }^{u} a \quad \forall a \in S, u \in T, y \in X .
$$

Condition (8.12) gives the base for the induction. Assume (8.13) holds for all $a \in S, y \in X$, and all $u \in T$, with $|u| \leq n$. Let $a \in S, y \in X$, and $u \in T,|u|=n+1$. Then $u=\alpha v, v \in T,|v|=n, \alpha \in Y$ and the following equalities hold in $U$.

$$
\begin{array}{rlrl}
u^{y} a=(\alpha v)^{y} a & \left.={ }^{\left(\alpha^{v} y\right.}\right)\left(v^{y}\right) a & & \text { : by MR2 } \\
& \left.={ }^{\left(\alpha^{v} y\right.}\right)\left({ }^{\left(v^{y}\right)} a\right) & \\
& ={ }^{\alpha}\left({ }^{v} a\right) & & \text { : by stu } 1 \text { and } \mathrm{IH} \\
& ={ }^{(\alpha v)} a={ }^{u} a . &
\end{array}
$$

This proves 8.130

Step 3. Finally, we prove (8.9), for all $a, b \in S, u \in T$, by induction on the length $|b|$ of $b$. The base of the induction is given by (8.13). Assume (8.9) holds for all $b \in S$, with $|b| \leq n$. Let $b=c y, c \in S,|c|=n, y \in X$.

$$
\begin{array}{rlrl}
u^{b} a={ }^{\left(u^{c y}\right)} a & ={ }^{\left(u^{c}\right)^{y} a} & \\
& ={ }^{\left(u^{c}\right)} a & & \text { : since } u^{c} \in T \text { and by IH } \\
& ={ }^{u} a & & \text { : by IH. }
\end{array}
$$

The remaining stu conditions, see (8.4), are proven by a similar argument. We have proven part (1).

Each of the parts (1) and (2) should be proved separately, although we use similar arguments, since, in general, the braided monoids $U, S, T$ are not embedded in the corresponding braided groups.

Sketch of proof of (2). Note that every element $a \in G$ can be presented as a monomial

$$
a=\zeta_{1} \zeta_{2} \cdots \zeta_{n}, \quad \zeta_{i} \in Z \bigcup Z^{-1} .
$$

By convention we consider a reduced form of $a$, that is a presentation 8.16 with minimal length $n$. Bearing this in mind, we prove (8.4) in $G_{Z}$, using an argument similar to our argument for monoids, but at each step we use induction on the length $n$ of the reduced form of the corresponding words $a, u, b$. 


\section{4. "Local" conditions sufficient for a generalized strong twisted unions} of non-degenerate braided sets to be also a braided set.

Definition 8.13. [19] Given a quadratic set $(X, r)$ we extend the actions ${ }^{x} \bullet$ and

- $x$ on $X$ to left and right actions on $X \times X$ as follows. For $x, y, z \in X$ we define:

$$
{ }^{x}(y, z):=\left({ }^{x} y,{ }^{x^{y}} z\right) \text {, and }(x, y)^{z}:=\left(x^{y} z, y^{z}\right) .
$$

The map $r$ is called, respectively, left and right invariant if

$$
\text { 12: } \quad r\left({ }^{x}(y, z)\right)={ }^{x}(r(y, z)), \quad \mathbf{r 2}: \quad r\left((x, y)^{z}\right)=(r(x, y))^{z}
$$

hold for all $x, y, z \in Z$.

Conditions $\mathbf{1 2}$ and $\mathbf{r} \mathbf{2}$ give a more compact way to express $\mathbf{1 1}, \mathbf{r} \mathbf{1}$, $\mathbf{l r} \mathbf{3}$, since the following implications hold:

$$
12 \Longleftrightarrow 11, \operatorname{lr} 3 ; \quad \mathrm{r} 2 \Longleftrightarrow \mathrm{r} 1, \operatorname{lr} 3 .
$$

Remark 8.14. [19] Let $(X, r)$ be a quadratic set. Then the following three conditions are equivalent: (a) $(X, r)$ is a braided set; (b) $(X, r)$ satisfies $\mathbf{1 1}$ and $\mathbf{r 2}$; (c) $(X, r)$ satisfies $\mathbf{r} \mathbf{1}$ and $\mathbf{1 2}$.

Notation 8.15. [19] When we study extensions it is convenient to have a 'local' notation for some of our conditions, in which the specific elements for which the condition is being imposed will be explicitly indicated in lexicographical order of first appearance. Thus for example $\mathbf{l} \mathbf{1}(\mathbf{x}, \mathbf{y}, \mathbf{z})$ means the condition exactly as written in Lemma 2.2 for the specific elements $x, y, z$. Similarly $\mathbf{r} 2(\mathbf{x}, \mathbf{y}, \mathbf{z})$ means for the elements $x, y, z$ exactly as appearing as in Definition 8.13

In this section we consider triples in the set $Z^{3}$ so for example

$$
\mathbf{1 1}(\mathbf{x}, \alpha, \mathbf{y}): \quad{ }^{x}\left({ }^{\alpha} y\right)={ }^{x} \alpha\left(x^{\alpha} y\right), \alpha, x, y \in Z .
$$

Finally, we use this notation to specify the restrictions of any of our conditions to subsets of interest. For example

$$
\begin{aligned}
& \mathbf{l}(\mathbf{X}, \mathbf{Y}, \mathbf{X}):=\{\mathbf{l 1}(\mathbf{x}, \alpha, \mathbf{y}), \quad \forall x, y \in X, \alpha \in Y\} . \\
& \mathbf{r 2}(\mathbf{X}, \mathbf{Y}, \mathbf{X}):=(r(x, \alpha))^{y}=r\left((x, \alpha)^{y}\right), \quad \forall x, y \in X, \alpha \in Y .
\end{aligned}
$$

The following result gives a necessary and sufficient condition so that a (general) quadratic set which is a strong twisted union $(Z, r)=\left(X, r_{1}\right) \natural^{*}\left(Y, r_{2}\right)$ of two disjoint braided sets is also a braided set.

Proposition 8.16. Suppose a non-degenerate and injective quadratic set $(Z, r)$ is a generalized strong twisted union of two disjoint 2-cancellative braided sets $\left(X, r_{X}\right)$ and $\left(Y, r_{Y}\right)$. Then $(Z, r)$ obeys $Y B E$ iff the following hold.

(1) Conditions (i) through (iv) in Corollary 8.11 are satisfied;

(2) The actions satisfy the following four "mixed" conditions

$$
\mathbf{1 1}(\mathrm{X}, \mathrm{Y}, \mathrm{X}), \mathbf{r 2}(\mathrm{X}, \mathrm{Y}, \mathrm{X}), \mathbf{l 1}(\mathrm{Y}, \mathrm{X}, \mathrm{Y}), \mathbf{r 2}(\mathrm{Y}, \mathrm{X}, \mathrm{Y})
$$

Proof. The proof is routine and an experienced reader may skip it.

Assume $(Z, r)$ obeys YBE. Then, by Remark 8.14 conditions $\mathbf{1 1}$ and $\mathbf{~ 2 2 , ~ ( a n d ~}$ r1 and 12) are satisfied for any triple $(a, b, c) \in Z^{3}$. In particular, the mixed conditions (8.18) hold, which proves (2). By assumption the braided set $(Z, r)$ is a strong twisted union $Z=X \natural^{*} Y$, so the hypothesis of Corollary 8.11 is satisfied, which implies (1). 
Assume now that (1) and (2) are satisfied. We have to show that $(Z, r)$ is a braided set. Recall that the YB-diagram starting with the triple $(a, b, c) \in Z^{3}$ shows that

$$
\begin{aligned}
r^{12} r^{23} r^{12}(a, b, c)=r^{23} r^{12} r^{23}(a, b, c) & \Longleftrightarrow \mathbf{r} \mathbf{1}(\mathbf{a}, \mathbf{b}, \mathbf{c}), \mathbf{1 2}(\mathbf{a}, \mathbf{b}, \mathbf{c}) \\
& \Longleftrightarrow \mathbf{1}(\mathbf{a}, \mathbf{b}, \mathbf{c}), \mathbf{r 2}(\mathbf{a}, \mathbf{b}, \mathbf{c}),
\end{aligned}
$$

$\forall a, b, c \in Z$.

There is nothing to prove if $(a, b, c) \in X^{3}$, or $(a, b, c) \in Y^{3}$, since by hypothesis $\left(X, r_{X}\right)$ and $\left(Y, r_{Y}\right)$ are braided sets.

Our argument uses the presentation of the set $Z^{3} \backslash\left(X^{3} \cup Y^{3}\right)$ as a union of six disjoint subsets

$$
\begin{aligned}
Z^{3} \backslash\left(X^{3} \cup Y^{3}\right)= & (X \times X \times Y) \bigcup(Y \times X \times X) \bigcup(X \times Y \times Y) \\
& \bigcup(Y \times Y \times X) \bigcup(X \times Y \times X) \bigcup(Y \times X \times Y) .
\end{aligned}
$$

Clearly, $(Z, r)$ obeys YBE iff each of the sets on the right-hand side of the above equality satisfies simultaneously the "mixed" conditions $\mathbf{1 1}$ and $\mathbf{r 2}$ (or equivalently, r1 and 12). Analyzing with details each of the corresponding six cases we note that

Condition (1) implies (a) $\mathbf{1 1}(\mathrm{X}, \mathrm{X}, \mathrm{Y})$ and $\mathbf{r 2}(\mathrm{X}, \mathrm{X}, \mathrm{Y})$; (b) $\mathbf{1 1}(\mathrm{Y}, \mathrm{X}, \mathrm{X})$, and r2(Y, $\mathrm{X}, \mathrm{X})$; (c) $\mathbf{1 1}(\mathrm{X}, \mathrm{Y}, \mathrm{Y})$, and $\mathbf{r 2}(\mathrm{X}, \mathrm{Y}, \mathrm{X})$; (d) $\mathbf{1 1}(\mathrm{Y}, \mathrm{Y}, \mathrm{X})$, and $\mathbf{r 2}(\mathrm{Y}, \mathrm{Y}, \mathrm{X})$. (In fact (1) encodes exactly these eight (mixed) conditions).

Condition (2) gives the missing "mixed" conditions (8.18) not encoded in (1).

Acknowledgments. This paper was written during my visits at the Abdus Salam International Centre for Theoretical Physics (ICTP), Trieste, Summers 2018, 2019, and at MPIM, Bonn in 2019. It is my pleasant duty to thank MPIM, Bonn and the Mathematics group of ICTP for the inspiring atmosphere.

\section{REFERENCES}

[1] N. Andruskiewitsch, And M. Graña, From racks to pointed Hopf algebras, Adv. in Math. 178.2 (2003) 177-243.

[2] M. Artin, W. Schelter, Graded algebras of global dimension 3, Adv. in Math. 66 (1987) 171216.

[3] G. M. Bergman, The diamond lemma for ring theory, Adv. in Math. 29 (1978) 178-218.

[4] J. S. Carter, M. Elhamdadi, M. Saito, Homology theory for the set-theoretic Yang-Baxter equation and knot invariants from generalizations of quandles, Fund. Math. 184 (2004), 31-54.

[5] Ferran Cedó, Tatiana Gateva-Ivanova and Agata Smoktunowicz, On the Yang-Baxter equation and left nilpotent left braces, J. Pure and Applied Algebra 221, (2017), 751-756.

[6] F. Cedó, T. Gateva-Ivanova, A. Smoktunowicz, Braces and symmetric groups with special conditions, J. of Pure and Applied Algebra 222, (2018) 3877-3890.

[7] F. Cedó, E. Jespers, J. Okninski, Retractability of set theoretic solutions of the Yang-Baxter equation, Adv. in Math. 224 (2010) 2237-2738.

[8] F. Chouraqui, An Algorithmic construction of group automorphisms and the quantum YangBaxter equation, Communications in Algebra 46.11 (2018), 4710-4723.

[9] V. G. Drinfeld, On some unsolved problems in quantum group theory, Quantum Groups (P. P. Kulish, ed.), Lect. Notes in Mathematics, vol. 1510, Springer Verlag, 1992, 1-8.

[10] P. Etingof, T. Schedler, A. Soloviev, Set-theoretical solutions to the quantum YangBaxter equation, Duke Math. J. 100 (1999) 169-209.

[11] Gateva-Ivanova, T., Noetherian properties of skew polynomial rings with binomial relations, Trans. Amer. Math. Soc. 343 (1994) 203-219.

[12] T. Gateva-Ivanova, Skew polynomial rings with binomial relations, J. Algebra 185 (1996) 710-753.

[13] T. Gateva-Ivanova, A combinatorial approach to the set-theoretic solutions of the YangBaxter equation, J.Math.Phys., 45 (2004) 3828-3858. 
[14] T. Gateva-Ivanova, Quantum binomial algebras, Artin-Schelter regular rings, and solutions of the Yang-Baxter equations, Serdica Math. J. 30 (2004) 431-470.

[15] T. Gateva-Ivanova, Garside structures on monoids with quadratic square-free relations, Algebr. Represent. Theor 14 (2011), 779-802.

[16] T. Gateva-Ivanova, Quadratic algebras, Yang-Baxter equation, and Artin- Schelter regularity, Advances in Mathematics, 230 (2012), 2152-2175.

[17] Tatiana Gateva-Ivanova, Set-theoretic solutions of the Yang-Baxter equation, Braces and Symmetric groups, Advances in Mathematics 338 (2018), 649-701.

[18] Tatiana Gateva-Ivanova, Peter Cameron, Multipermutation solutions of the Yang-Baxter equation, Comm. Math. Phys, 309 (2012), 583-621.

[19] T. Gateva-Ivanova, S. Majid , Matched pairs approach to set theoretic solutions of the Yang-Baxter equation, J. Algebra 319 (2008) 1462-1529.

[20] T. Gateva-Ivanova, S. MajID, Quantum spaces associated to multipermutation solutions of level two, Algebr. Represent. Theor., 14 (2011) 341-376

[21] T. Gateva-Ivanova, M. Van den Bergh, Semigroups of I-type, J. Algebra 206 (1998) 97112.

[22] M. Grana, I. Hechenberger and L. Vendramin , Nichols algebras of group type with many quadratic relations, Adv. in Math. 227 (2011) 1956-1989.

[23] L. Guarnieri and L. Vendramin, Skew braces and the Yang-Baxter equation, Math. Comp. 86307 (2017) 2519-2534.

[24] V. Lebed and L. Vendramin, On structure groups of set-theoretic solutions to the YangBaxter equation, Proceedings of the Edinburgh Mathematical Society, 62 (3), 683-717.

[25] J. Lu, M. Yan, Y. Zhu, On the set-theoretical Yang-Baxter equation, Duke Math. J. 104 (2000) 1-18.

[26] S. MAJID, Foundations of the Quantum Groups, Cambridge University Press, 1995. $[24]$

[27] N.Yu. Reshetikhin, L.A. Takhtadzhyan, L.D. Faddeev, Quantization of Lie groups and Lie algebras, Algebra i Analiz 1 (1989) 178-206 (in Russian); English translation in: Leningrad Math. J. 1 (1990) 193-225.

[28] Yu. I. MAnin, Some remarks on Koszul algebras and quantum groups, Ann. de L'Inst. Fourier, 37 (1987) 191-205.

[29] YU. I. MANIN, Quantum groups and non-commutative geometry, Les publications CRM, Université de Montreal (1988).

[30] S. Nelson, Classification of finite Alexander quandles, Proceedings of the Sping Topology and Dynamical Systems Conference.Topology Proc. 27 (2003) 245-258.

[31] A. Polishchuk, L. Positselski, Quadratic Algebras, University Lecture Series, v.37 American Mathematical Society, 2005.

[32] St. Priddy, Koszul resolutions, Trans. Amer. Math. Soc. 152 (1970) 39-60.

[33] W. Rump, A decomposition theorem for square-free unitary solutions of the quantum YangBaxter equation, Advances in Mathematics, 193 (2005) 40-55.

[34] A. Smoktunowicz and L. Vendramin, On skew braces (with an appendix by N. Byott and L. Vendramin, J. Comb. Algebra 21 (2018) 47-86.

[35] A. Soloviev, Non-unitary set-theoretic solutions to the quantum Yang-Baxter equation, Math. Res. Lett., 75-6 (2000) 557-596.

[36] M. TAKeuchI, Survey on matched pairs of groups. An elementary approach to the ESS-LYZ theory, Banach Center Publ. 61 (2003) 305-331.

[37] L. Vendramin, Extensions of set-theoretic solutions of the Yang-Baxter equation and a conjecture of Gateva-Ivanova, J. Pure Appl. Algebra, 220 (2016) 2064-2076 .

American University in Bulgaria, 2700 Blagoevgrad, Bulgaria

E-mail address: tatyana@aubg.edu 